\title{
Multifragmentation near the threshold
}

\author{
A. Bohnet and J. Aichelin* \\ Institut für Theoretische Physik, Universität Heidelberg, Philosophenweg 19, 6900 Heidelberg, Germany \\ and Max-Planck-Institut für Kernphysik, 6900 Heidelberg, Germany \\ J. Pochodzalla and W. Trautmann \\ Gesellschaft für Schwerionenforschung, 6100 Darmstadt, Germany \\ G. Peilert, H. Stöcker, and W. Greiner \\ Institut für Theoretische Physik, J. W. Goethe-Universität, 6000 Frankfurt am Main, Germany
}

(Received 24 July 1990)

\begin{abstract}
We investigate the onset of multifragmentation employing an improved version of the $N$-body "quantum" molecular-dynamics approach. We study in detail the reaction ${ }^{18} \mathrm{O}+{ }^{197} \mathrm{Au}$ at $84 \mathrm{MeV} / \mathrm{nucleon}$ and find good agreement between the calculated results and the data for the double-differential proton cross section, the mass yield, the multiplicity, the kinetic energy of the fragments, and even for the kinematic correlations between intermediate mass fragments (IMF's), which have been measured in this experiment for the first time. We observe a strong correlation between the impact parameter and both the size of the target remnant as well as the average proton multiplicity. Hence both observables can be used to determine the impact parameter experimentally. The IMF's come from the most central collisions. The calculations confirm the experimental result that they are not emitted from an equilibrated system. Although the inclusive energy spectra look thermal, we cannot identify an impact parameterindependent isotropically emitting source. Even in central collisions global equilibrium is not observed. We find that multifragment emission at this bombarding energy is caused by a process very similar to that proposed in the macroscopic cold multifragmentation model. Thus it has a different origin than at beam energies around $1 \mathrm{GeV} /$ nucleon, although the mass yield has an almost identical slope.
\end{abstract}

\section{INTRODUCTION}

Intermediate-energy heavy-ion collisions in the energy regime between 30 and $200 \mathrm{MeV} /$ nucleon are not well understood presently. This is due to the observation that between these energies a transition takes place between the typical low-energy and the typical high-energy reaction mechanisms. At lower energies the central collision data are compatible with the assumption that all projectile and target nucleons form a compound nucleus [1]. It decays by sequential emission of light particles $(\gamma, n, p, \alpha, \ldots)$ and intermediate-mass fragments (IMF's). The formation and decay of the compound nucleus are completely independent. Thus the emitted particles do not show any correlation with the entrance channel. At higher energies the gross features of the reaction are approximately described in the participant-spectator model [2]. The participants are those nucleons which are located in the geometrical overlap of projectile and target. The rest are called spectators. If the participants come to equilibrium in the course of the reaction and form a high-temperature gas of nucleons and light fragments, the fireball model emerges. The fireball model has been successfully applied to describe proton and neutron spectra at the Bevalac energies.

Thus these models seem to indicate equilibration at low and high energies. The proposed mechanism which leads to the equilibration of the system is completely different, however. At low energy, $E_{\text {kin }} \ll 30 \mathrm{MeV} /$ nucleon, few collisions together with the time-dependent mean field are sufficient to randomize the beam energy of the projectile nucleons. They are not able to leave the system after having traversed the target nucleus, but are trapped for quite a long time. This time is sufficient to populate all available states with the same probability. Almost all energetically possible nucleon-nucleon collisions are blocked because of the occupation of the final-state phase space by other particles of the system. Thus the equilibration is accomplished by the potential and not by collisions [i.e., by the real part $(\operatorname{Re} G)$ and not by the imaginary part $\left(\operatorname{Im} G \propto \sigma_{\text {tot }}\right)$ of the Brückner $G$ matrix] [3]. In the vacuum the potential term disappears and only the scattering terms are present. At much higher energies the situation is quite opposite. The stopping effect of the mean field is almost negligible and the equilibration (which is, however, not complete) is caused by two-body collisions. They are much less suppressed by the Pauli blocking as compared to lower beam energies: The total available phase space is larger, and therefore the average occupation of the phase space by other nucleons is lower [4-6].

For asymmetric collisions in the intermediate-energy regime (between 30 and $200 \mathrm{MeV} /$ nucleon), the following scenario is plausible: The few allowed collisions neither slow down the projectile nucleons sufficiently to be trapped in the target nor are they frequent enough to form an equilibrium system with the target participants. Thus nonequilibrium features are expected to appear, 
which make this energy regime quite interesting, but also quite complicated to understand [4-9].

The first experiments performed in this energy regime at CERN [10-21] and Michigan State University [22-25] indeed have shown that there exists no single source of isotropic emission, neither for protons nor for IMF's. In the projectile-target c.m. system, we always observe an enhancement of the total cross section in the forward direction [15]. This enhancement is correlated with a higher average energy in the forward direction. It is expected from the fireball model that different impact parameters lead to different longitudinal momentum transfers and hence to different source velocities and temperatures. A multisource fit usually describes the singleparticle spectra reasonably well $[22,23]$. One may question, however, whether these fits are merely a parametrization of the data or whether this finding presents evidence for an equilibration of the emitting participant source.

With the advent of the Boltzmann-UehlingUhlenbeck-Vlasov-Uehling-Uhlenbeck (BUU/VUU) calculations, it became possible to simulate the proton and deuteron spectra in heavy-ion reactions from 20 $\mathrm{MeV} /$ nucleon to $1 \mathrm{GeV} /$ nucleon [4-9]. The unexpected good agreement between the predicted and measured spectra gave confidence that the essential physics is contained in this approach. Calculations showed a strong impact-parameter dependence of both the transverse momentum transfer and longitudinal momentum transfer, which was never found to be complete in asymmetric reactions [5,6]. An isotropically emitting source could be identified only at the end of the reaction [5]. The number of nucleons emitted from that source is small as compared to the total number of emitted protons. Most nucleons escape before equilibration, and their emission pattern depends strongly on the number of collisions they suffer while traversing the target [5]. Thus BUU/VUU calculations did not support a clear distinction between preequilibrium and equilibrium emission as requested by thermal models. Rather, they showed a quite smooth transition.

While the BUU calculations elucidated the physical origin of the shape of the proton spectra, they do not allow to investigate the origin of IMF's because of the limitation of their predictive power to one-body observables. For a quantitative comparison with experiment, it is necessary to treat the IMF's properly. Already, below $100 \mathrm{MeV} /$ nucleon, events with up to four IMF's were observed [26]. Emulsion experiments have shown that the number of IMF's in a central collision of a light projectile with a heavy target increases continuously from 25 to 200 $\mathrm{MeV} /$ nucleon [27].

The theoretical models applied so far to describe the production of IMF's at this energy are either phenomenological and macroscopic - for a review we refer to Ref. [27]-or start with the assumption that equilibrium is achieved before fragment emission $[1,28,29]$.

The macroscopic models assume that in asymmetric collisions the IMF's are made of spectator matter [30,31]. By nucleon-nucleon collisions some participant nucleons gain sufficient transverse momentum so that they enter the spectator matter. Their interaction with the spectators destabilizes the spectator matter. This causes a cold breakup of the spectator matter into many fragments.

The thermal models can be further subdivided: (a) The compound nucleus model assumes a sequential decay on a time scale which allows equilibration of the whole $[1,29]$ or of a part [28] of the system in between subsequent evaporation steps. (b) The statistical models [32-34] assume an instantaneous decay.

Both thermal models are only compatible with highenergy fragmentation data of asymmetric heavy-ion reactions if one argues in favor of a two-step process. In the first step fast preequilibrium particles are emitted-this explains the anisotropy of the proton spectra-whereas in the second step an equilibrated system which contains all spectators and some of the participants is the source of the emission of fragments. Because also the doubledifferential cross section of IMF's [15] is not isotropic, these models employ either several sources-usually a projectile, a target, and a participant source-or introduce impact-parameter-dependent source velocities and temperatures whose functional dependence is treated as a fit because there exist no data which allow us to estimate the momentum and energy carried away by preequilibrium particles.

Recently, it has been argued that both kinds of thermal models predict a different distribution of relative velocities between IMF's [29]. Thus, if thermal models were applicable, the mechanism could be determined experimentally. A careful investigation of the experimental results which includes the recently measured kinetic-energy spectra of the heavy residues rules out the possibility that the IMF's are either emitted from an excited compound nucleus or created in a statistical breakup of the system. This we will show later.

If the system does not reach equilibrium, it is unavoidable to investigate the dynamics of the reaction in a model which includes also information about fragments. Such a model, the quantum molecular-dynamics (QMD) model, which describes the complete time evolution of the system from the initial separation of projectile and target to the final distribution of nucleons and fragments, was advanced over the last years $[35,36]$. Up to now, the model was used to describe fragment formation at beam energies larger than $200 \mathrm{MeV} /$ nucleon [35]. Here we report on a detailed comparison with experiment for the reaction $84 \mathrm{MeV} /$ nucleon ${ }^{18} \mathrm{O}+{ }^{197} \mathrm{Au}[10-19]$. For this purpose we had to improve our model.

The aim of this paper is twofold: First, we will demonstrate that all experimental data which can be calculated in the QMD approach agree quite well with experiment. This is especially true for those observables which rule out the applicability of equilibrium models. Thus, even near the threshold, multifragmentation can be embedded in a model which also has been proven to describe the highly nonthermal single-particle spectra. There is one exception, the projectilelike fragments which are measured in coincidence with fast protons in very peripheral reactions appear at $\vartheta \approx-5^{\circ}$ instead of $\vartheta \approx 5^{\circ}$ as seen in experiment. Then we will take advantage of the fact that, in QMD calculations, not only the momenta, but also all 
positions are recorded. This allows us to investigate not only the reaction mechanism in detail, but also the impact-parameter dependence of different observables. Thus a detailed understanding of these intermediateenergy heavy-ion reactions is possible and experimental signatures for different impact parameters can be proposed. This is the second aim of this paper. The impact-parameter dependence of a variety of observables has been studied for similar reactions $(84 \mathrm{MeV} /$ nucleon $\mathrm{C}+\mathrm{Au}$ [5] and $90 \mathrm{MeV} /$ nucleon $\mathrm{O}+\mathrm{Au}$ [37]) in the BUU approach. Both calculations are compatible with each other and give reasonable results as far as protons are concerned. They fail badly, as expected, for target or intermediate-mass fragment observables because the BUU theory as a one-body theory is not designed to describe the fragmentation of the target nucleus. Thus the prediction of the linear momentum transfer to the target nucleus and the distribution of masses of the target remnant are not in agreement with experiment and the impact-parameter dependence of the mass of the heavy residue is rather flat. Even in central collisions the remnant has a mass of about $A=188$, whereas in experiments target remnants of $A=150$ have been observed. This requires a 5 times larger mass loss as predicted by BUU.

In Sec. II we will describe in detail the improvements of the QMD approach (code version 102) necessary to describe reactions in this energy regime. They include the motion of projectile and target along Coulomb trajectories before the nuclei touch and the introduction of a Yukawa potential, which dampens the density fluctuations and stabilizes small fragments. We will report on the tests performed and will show that nuclei in the QMD model have the proper binding energy and can be kept stable for a sufficiently long time span to describe intermediate-energy heavy-ion reactions. Section III is devoted to an overview of the reaction, a detailed investigation of the impact-parameter dependence of some observables, and to the comparison with the experiment. In Sec. IV we will investigate why nuclei fragment into many pieces even at this low energy. Finally, in Sec. V we will present our conclusions.

\section{MODEL}

In this section we describe the details of the QMD model and its numerical realization [35]. We give an account of the tests performed and show how different potentials change the stability of the nuclei.

The typical time for a heavy-ion reaction, as we will see, is around $200 \mathrm{fm} / c$. For this time noninteracting nuclei have to be stable. Otherwise, one cannot be sure that the results really reveal the physics and are not just numerical artifacts. The stability, and hence the successful simulation of heavy-ion collisions, depends on the solution of two critical problems: the choice of the initial configuration and the propagation of the $A_{T}+A_{P}$ system. We start with the first topic.

\section{A. Initialization}

When we compared quantal time-dependent HartreeFock (TDHF) and classical (Vlasov) mean-field systems, we found an almost identical time evolution of the nuclear density for beam energies larger than 25 $\mathrm{MeV} /$ nucleon [38]. This means, first of all, a justification for terminating the $\hbar$ expansion of the potential term in the time-evolution equation [3]. Nevertheless, this is quite surprising because of the different initializations [38]. From these results we have concluded that, at for bombarding energies larger than $25 \mathrm{MeV} /$ nucleon, the detailed form of the wave functions has only a minor influence on the time evolution of the bulk properties of the system, especially on the single-particle observables, if they fulfill minimal requirements. The observables are determined by the single-particle density and are rather independent of the way the single-particle density is generated by the density distributions of the individual nucleons. We cannot expect to learn much about twonucleon correlations from these different theories. Therefore, if one wants to construct an $N$-body theory, one has to start with an educated guess for the $N$-body Wigner density [3]. The description of the nuclear wave function as a product of $N$ coherent states,

$$
\psi_{i}\left(\mathbf{r}, \mathbf{p}_{i 0}, \mathbf{r}_{i 0}, t\right)=\frac{1}{[2 \pi L(t)]^{3 / 4}} e^{-\left[\mathbf{r}-\mathbf{r}_{i 0}(t)\right]^{2} / 4 L(t)} e^{i \mathbf{p}_{i 0}(t) \mathbf{r}},
$$

allows one to fulfill most of the experimental and theoretical demands on single-particle distributions and twobody correlations. (Above and in what follows, we set $\hbar=1)$. The Wigner transform of the coherent states is Gaussian in momentum and coordinate space and is given by

$$
\begin{array}{r}
f_{i}(\mathbf{r}, \mathbf{p}, t)=\frac{1}{(2 \pi)^{3}} \int e^{-i \mathbf{p} \mathbf{r}_{12}} \psi_{i}\left[\mathbf{r}+\frac{\mathbf{r}_{12}}{2}, t\right] \\
\quad \times \psi_{i}^{*}\left[\mathbf{r}-\frac{\mathbf{r}_{12}}{2}, t\right) d^{3} r_{12} \\
=\frac{1}{\pi^{3}} e^{-\left[\mathbf{r}-\mathbf{r}_{i 0}(t)\right]^{2} / 2 L} e^{-\left[\mathbf{p}-\mathbf{p}_{i 0}(t)\right]^{2} 2 L}
\end{array}
$$

The Wigner representation of our Gaussian wave packets obeys the uncertainty relation $\Delta r_{n} \Delta p_{m}=\frac{1}{2} \delta_{n m}$.

Our $N$-body Wigner densities $f^{(N)}$ is the direct product of the Wigner densities of $N$ coherent states. The width of the Gaussians has the fixed value $L=1.08 \mathrm{fm}^{2}$. This corresponds to a root-mean-square radius of the nucleons of $1.8 \mathrm{fm}$.

The one-body densities in coordinate and momentum space are

$$
\begin{aligned}
\rho(\mathbf{r}, t)= & \sum_{i=1}^{N} \int \delta\left(\mathbf{r}-\mathbf{r}_{i}\right) f^{(N)}\left(\mathbf{r}_{1}, \ldots \mathbf{r}_{N}, \mathbf{p}_{1}, \ldots \mathbf{p}_{N}, t\right) \\
& \times d^{3 N} p d^{3 N} r \\
= & \sum_{i=1}^{N} \frac{1}{(2 \pi L)^{3 / 2}} e^{-\left[\mathbf{r}-\mathbf{r}_{i 0}(t)\right]^{2} / 2 L}
\end{aligned}
$$

and, equivalently 


$$
\begin{aligned}
g(\mathbf{p}, t)=\sum_{i=1}^{N} \int & \delta\left(\mathbf{p}-\mathbf{p}_{i}\right) f^{(N)}\left(\mathbf{r}_{1}, \ldots, \mathbf{r}_{N}, \mathbf{p}_{1}, \ldots, \mathbf{p}_{N}, t\right) \\
& \times d^{3 N} r d^{3 N} p
\end{aligned}
$$

Next, we have to determine the centroids $\mathbf{r}_{i 0}$ and $\mathbf{p}_{i 0}$ of the Gaussians. A random choice of the $A_{T}+A_{P}$ centroids in coordinate and momentum space, where the momentum is chosen between zero and the local Fermi momentum and coordinates are distributed in a sphere of radius $R=r_{0} A^{1 / 3}$, is not sufficient to maintain the stability of the nuclei for the required time span. Because of fluctuations, a limited sequence of random numbers does not create the ground state of a nucleus, but rather a metastable excited state which decays by emission of nucleons. The time span for which the nucleus has to be stable implies an upper limit to the excitation energy which can be tolerated.

Eigenstates of a Hamiltonian have to fulfill the uncertainty relation; i.e., each level fills a volume of $h^{3}$ in phase space. If a system is in the ground state, the phase space is densely filled up to a maximum value in coordinate and momentum space. Loosely speaking, there is no hole in the phase space. It is this property of the ground state which we employ to initialize the nuclei. First, we determine the position of the nucleons in a sphere of the radius $r=1.2 A^{1 / 3}$. We draw random numbers, but reject those which would position the centers of two nucleons closer than $r_{\min }=1.5 \mathrm{fm}$. The next step is to determine the local potential $U(r)$ generated by all the other nucleons at the centers of the Gaussians. The local Fermi momentum we determine by the relation $p_{F}\left(r_{i 0}\right)=\sqrt{2 m U\left(r_{i 0}\right)}$, where $U\left(r_{i 0}\right)$ is the potential energy of the particle $i$. Finally, the momenta of all particles are chosen randomly between zero and the local Fermi momentum. We then reject all random numbers which yield two particles closer in phase space than $\left(\mathbf{r}_{i 0}-\mathbf{r}_{j 0}\right)^{2}\left(\mathbf{p}_{i 0}-\mathbf{p}_{j 0}\right)^{2}=d_{\min }$. Typically only 1 out of 50000 initializations is accepted under the present criteria. This procedure ensures that the nuclei have the proper rms radii in coordinate and momentum space.

\section{B. Propagation in the effective potential}

Successfully initialized nuclei are boosted toward each other with proper c.m. velocity using relativistic kinematics. The centers of the distribution are moving along Coulomb trajectories with the assumption that all charges are located at the centers of the nuclei. The distribution is kept fixed until the distance of the surfaces of the nuclei is $2 \mathrm{fm}$. From then on, the centroids of the Gaussians $\left(\mathbf{r}_{i 0}, \mathbf{p}_{i 0}\right)$ are propagated under the influence of mutual two- and three-body interactions as described by the Poisson brackets

$$
\dot{\mathbf{r}}_{i 0}=\left\{\mathrm{r}_{i 0}, \sum_{j} H_{j}\right\}=\left\{\mathbf{r}_{i 0}, T+U_{i}\right\}
$$

and

$$
\dot{\mathbf{p}}_{i 0}=\left\{\mathbf{p}_{i 0}, \sum_{j} H_{j}\right\}=\left\{\mathbf{p}_{i 0}, T+U_{i}\right\} .
$$

$H_{j}$ is the total energy of particle $j, T$ is the total kinetic energy, and $U_{i}$ is the potential of particle $i$. These differential equations are solved using an Eulerian integration routine with a fixed time step $\Delta t$ :

$$
\begin{aligned}
\mathbf{p}_{i 0}(n+1)= & \mathbf{p}_{i 0}(n)-\nabla_{r_{i 0}} U_{i}\left(n+\frac{1}{2}\right) \Delta t, \\
\mathbf{r}_{i 0}\left(n+\frac{1}{2}\right)= & \mathbf{r}_{i 0}\left(n-\frac{1}{2}\right)+\frac{\mathbf{p}_{i 0}(n)}{\left[p_{i 0}^{2}(n)+m_{i}^{2}\right]^{1 / 2}} \Delta t \\
& +\nabla_{p_{i 0}} U_{i}\left(n-\frac{1}{2}\right) \Delta t .
\end{aligned}
$$

\section{Static interactions}

We replace the real part of the transition or the Brückner $G$ matrix by local Skyrme-type interactions, supplemented by a long-range Yukawa interaction, which is necessary to reproduce the surface, and an effectivecharge Coulomb interaction, where all particles of projectile and target have a charge $Z_{P} / A_{P}$ and $Z_{T} / A_{T}$, respectively.

Our total static interaction reads

$$
V=V^{\mathrm{loc}}+V^{\mathrm{Yuk}}+V^{\mathrm{Coul}} \text {, }
$$

where the different terms are

$$
V^{\mathrm{loc}}=t_{1} \delta\left(\mathbf{r}_{1}-\mathbf{r}_{2}\right)+t_{2} \delta\left(\mathbf{r}_{1}-\mathbf{r}_{2}\right) \delta\left(\mathbf{r}_{1}-\mathbf{r}_{3}\right)
$$

and

$$
V^{\mathrm{Yuk}}=t_{3} \frac{e^{-\left|\mathbf{r}_{1}-\mathbf{r}_{2}\right| / m}}{\left|\mathbf{r}_{1}-\mathbf{r}_{2}\right| / m},
$$

with $m=1.5 \mathrm{fm}$ and $t_{3}=-6.66 \mathrm{MeV}$. These parameters give the best preservation of the nuclear surface, as we will see in Sec. II C 1.

The total energy $H_{i}$ of the particle $i$ is the sum of the kinetic and potential energies:

$$
H_{i}=T_{i}+\frac{1}{2} \sum_{j \neq i} U_{i j}^{(2)}+\frac{1}{3} \sum_{\substack{j, k ; j \neq k ; \\ j, k \neq i}} U_{i j k}^{(3)} .
$$

$T_{i}$ refers to the kinetic energy of particle $i$, and the potentials $U^{(2)}$ and $U^{(3)}$ are defined as

$$
\begin{aligned}
U_{i j}^{(2)}=\int & V^{(2)}\left(\mathbf{r}_{i}-\mathbf{r}_{j}\right) f_{i}\left(\mathbf{r}_{i}, \mathbf{p}_{i}, t\right) f_{j}\left(\mathbf{r}_{j}, \mathbf{p}_{j}, t\right) d^{3} r_{i} d^{3} r_{j} \\
& \times d^{3} p_{i} d^{3} p_{i}, \\
U_{i j k}^{(3)}=\int & V^{(3)}\left(\mathbf{r}_{i}, \mathbf{r}_{j}, \mathbf{r}_{k}\right) \prod_{v=i, j, k} f_{v}\left(\mathbf{r}_{v}, \mathbf{p}_{v}, t\right) d^{3} r_{v} d^{3} p_{v} .
\end{aligned}
$$

$V^{(2)}$ and $V^{(3)}$ are the two- and three-body parts of the interaction $V$ defined in Eq. (9). 
Performing the integration, one sees immediately that the local part of $\Sigma U_{i j}^{(2)}$ can be written as

$$
\sum_{j} U_{i j}^{(2) l o c}=t_{1} \widetilde{\rho}\left(\mathbf{r}_{i 0}\right)
$$

where the interaction density $\widetilde{\rho}\left(\mathbf{r}_{i 0}\right)$ is

$$
\widetilde{\rho}\left(\mathbf{r}_{i 0}\right)=\frac{1}{(4 \pi L)^{3 / 2}} 6 \sum_{j} e^{-\left(\mathbf{r}_{i 0}-\mathbf{r}_{j 0}\right)^{2} / 4 L} .
$$

The interaction density has twice the width of the singleparticle density and depends on the distance of the centroids. We can approximate $U^{(3)}$ as a function of $\widetilde{\rho}\left(\mathbf{r}_{i 0}\right)$ :

$$
\begin{aligned}
\sum_{j, k} U_{i, j, k}^{(3)} & =\frac{t_{3}}{(2 \pi L)^{3} 3^{3 / 2}} \sum_{\substack{j, k ; j \neq k ; \\
j, k \neq i}} \exp \left\{\left[\left(\mathbf{r}_{k 0}-\mathbf{r}_{i 0}\right)^{2}+\left(\mathbf{r}_{k 0}-\mathbf{r}_{j 0}\right)^{2}+\left(\mathbf{r}_{j 0}-\mathbf{r}_{i 0}\right)^{2}\right] / 6 L\right\} \\
& \approx \frac{t_{2}}{(2 \pi L)^{3} 3^{3 / 2}} \sum_{\substack{j, k ; j \neq k ; \\
j, k \neq i}} \exp \left\{\left[\left(\mathbf{r}_{i 0}-\mathbf{r}_{j 0}\right)^{2}+\left(\mathbf{r}_{i 0}-\mathbf{r}_{k 0}\right)^{2}\right] / 4 L\right\} \\
& =\frac{t_{2}(4 \pi L)^{3 v / 2}}{(2 \pi L)^{3(v-1) / 2}(v+1)^{3 / 2}} \widetilde{\rho}_{i}^{v}\left(\mathbf{r}_{i 0}\right),
\end{aligned}
$$

with $v=2$. This equation is used in the present calculation. However, it does not mean that there are strong, true-body interactions. In spin-saturated nuclear matter, the three-body interaction can either be viewed as a genuine three-body interaction or as the density dependence of the two-body interaction due to the hard core [39]. The Yukawa part of the potential energy is given by

$$
\sum_{j} U_{i j}^{(2) \mathrm{Yuk}}=t_{3} \sum_{j \neq i} \frac{L / m^{2}}{r_{i j} / 2 m}\left\{e^{-r_{i j} / m}\left[1-\Phi\left(x_{-}\right)\right]-e^{r_{i j} / m}\left[1-\Phi\left(x_{+}\right)\right]\right\},
$$

where $\Phi$ is the error function and its arguments are

$$
x_{ \pm}=\frac{\sqrt{L}}{m} \pm \frac{r_{i j}}{2 \sqrt{L}} \text {. }
$$

In the two equations above, $r_{i j}$ is the distance between the centers of the particles $i$ and $j$.

Next, we have to determine the parameters $t_{1}$ and $t_{2}$. We start from the observation that in nuclear matter, where the density is constant, the interaction density coincides with the single-particle density, and $U^{(2) l o c}$, as well as $U^{(2) \text { Yuk }}$, is directly proportional to $\rho / \rho_{0}$, where $\rho_{0}$ is the normal nuclear-matter density. The three-body part $U^{(3)}$ of the interaction is proportional to $\left(\rho / \rho_{0}\right)^{2}$. If we adopt for $U^{(3)}$ the approximation (17), we can directly relate our parameters to nuclear-matter properties. In nuclear matter our Skyrme-like potential has the form

$$
U^{\mathrm{loc}}=\alpha \frac{\rho}{\rho_{0}}+\beta\left(\frac{\rho}{\rho_{0}}\right)^{2}
$$

which has two free parameters $\alpha$ and $\beta$ which can be fixed by the requirement that at normal nuclear-matter density the average binding energy is $-15.75 \mathrm{MeV}$ and the total energy has a minimum at $\rho_{0}$. The adjustment of the two parameters fixes the compressibility as well. One can generalize the potential to

$$
U^{\mathrm{loc}}=\alpha \frac{\rho}{\rho_{0}}=\beta\left(\frac{\rho}{\rho_{0}}\right)^{\gamma}
$$

We now have an additional third parameter $\gamma$, which allows us to fix the compressibility independently from the other quantities. This generalization can be translated back to the nucleon-nucleon potential in a unique way by identifying $v$ in Eq. (17) with $\gamma$. By varying these three parameters, we can investigate how different compressibilities, i.e., different equations of state, influence the observables.

The parameter $\alpha$ contains contributions from the local two-body potential as well as from the Yukawa potential. In nuclear matter there is no difference between local and nonlocal potentials. We can always expand nonlocal interactions such as the Yukawa interaction:

$$
\begin{aligned}
U_{i j}^{(2) \mathrm{Yuk}} & =t_{3} \int d^{3} r \int d^{3} r^{\prime} \frac{e^{-\left|\mathbf{r}-\mathbf{r}^{\prime}\right| / m}}{\left|\mathbf{r}-\mathbf{r}^{\prime}\right| / m} \rho_{i}(r) \rho_{j}\left(r^{\prime}\right) \\
& =4 \pi m^{3} t_{3} \int d^{3} r \rho_{i}(r) \rho_{j}(r)+O\left(\nabla^{2} \rho\right) \\
& =\frac{4 \pi m^{3} t_{3}}{(4 \pi L)^{3 / 2}} e^{-\left(\mathbf{r}_{i 0}-\mathbf{r}_{j 0}\right)^{2} / 4 L}+O\left(\nabla^{2} \rho\right)
\end{aligned}
$$

Thus, in nuclear matter, any combination of $t_{1}, t_{3}$, and $m$ is equivalent as long as $t_{1}-4 \pi m^{3} t_{3}=$ const.

This is not the case with finite nuclei. It turns out that (12) in the approximation (17) with the values for $t_{1}$ and $t_{2}$ obtained for the desired nuclear-matter properties gives about the right binding energy for finite nuclei also. Consequently, if we want to employ a Yukawa potential with parameters $t_{3}$ and $m$, we calculate its contribution to the potential energy of the particle $i$, which is then subtracted from the local two-body term by changing the coefficient $t_{1}$ to $t_{1 i}$ :

$$
t_{1 i} \widetilde{\rho}\left(\mathbf{r}_{i 0}\right)=t_{1} \widetilde{\rho}\left(\mathbf{r}_{i 0}\right)-\sum_{j} U_{i j}^{(2) \mathrm{Yuk}}
$$

Thus the total potential energy remains constant, in- 
dependent of the parameters of the Yukawa term we choose. The forces which determine the time evolution, however, depend strongly on the choice of the parameters.

For the actual propagation, we would like to stress that the explicit two- and three-body potentials [Eqs. (13), (17), and (18)] are used and not the nuclear-matter potentials [Eq. (21)]. This is important since the equivalence of both is only true in nuclear matter, not in finite nuclei.

The parameters of the static potential we use here are $\alpha=-356 \mathrm{MeV}, \beta=303 \mathrm{MeV}$, and $\gamma=\frac{7}{6}$. This set of parameters is usually called the "soft equation of state" and has an incompressibility constant of $200 \mathrm{MeV}$.

\section{Collisions}

The scattering of nucleons in nuclear matter in the low-density expansion should be described in terms of the Brückner $G$ matrix:

$$
G(E)=V+V \frac{Q}{E-e+i \varepsilon} G(E),
$$

where the Pauli operator $Q$ projects on unoccupied states and $e$ is the energy of the intermediate state, $e=p_{1}^{2} / 2 m+p_{2}^{2} / 2 m+U\left(p_{1}\right)+U\left(p_{2}\right)$. At high energies the influence of the Pauli blocking is small and the kinetic energy is large as compared to the Hartree-Fock potential $U$. Then the $G$ matrix becomes identical to the transition matrix which describes the scattering between two free nucleons. We assume for the time being that we can neglect the Pauli blocking of the intermediate states and include the Pauli blocking of the final state only. Of course, it would be highly desirable to have true inmedium-corrected scattering amplitudes. At high energies these are only available for an equilibrated environment and amount to a $30 \%$ reduction of the free cross section $\sigma_{\text {free }}[40,41]$. Recently, however, also an increase of the in-medium cross section compared to the free one has been put forward $[42,43]$. Thus, presently, it is hard to judge the direction of in-medium corrections to the nucleon-nucleon cross section on the basis of nuclearmatter calculations. At low energies the influence of the Pauli blocking of the intermediate states in the highly nonthermal environment at the beginning of a heavy-ion collision has been found to be small [44]. Thus we performed the calculation presented here with an isotropic 40-mb cross section, which requires less computer time than the calculation with the $G$-matrix cross section, but yields almost identical results.

\section{Pauli blocking of the final states}

Whenever a collision occurs, we check the phase space around the final states of the scattering partners. For simplicity, we assume that each nucleon occupies a sphere in coordinate and momentum space. This prescription yields the same Pauli-blocking ratio as an exact calculation of the overlap of the Gaussians, but is much less time consuming to calculate. We determine which fractions, $P_{1}$ and $P_{2}$, of the final phase spaces for each of the two scattering partners are already occupied by other nucleons. The collision is then allowed with a probability

$$
P_{\text {allowed }}=\left[1-\min \left(P_{1}, 1\right)\right]\left[1-\min \left(P_{2}, 1\right)\right],
$$

and, correspondingly, is blocked with the probability $\left(1-P_{\text {allowed }}\right)$. Whenever a collision is blocked, we replace the momentum of the scattering partners by the value it had prior to scattering. Care is taken for nucleons which are close to the surface of the many-nucleon system, where the above description includes also portions of phase space which are classically forbidden as a consequence of energy conservation. For a nucleus in its ground state, where all collisions should be blocked, we obtain an averaged blocking probability $\left\langle P_{\text {block}}\right\rangle$ of 0.96 . This determines the low-energy limit of our theory: Aiming at no more than $25 \%$ artificial collisions, i.e., collisions which are due to the imperfection of the Pauliblocking description, we can only admit beam energies at which no more than $84 \%$ of the collisions are blocked. Therefore, $E_{\text {lab }}=20 \mathrm{MeV} /$ nucleon is at the moment the lower bound for the range of validity of our approach.

\section{Numerical tests}

\section{Stability}

One basic requirement that the model has to fulfill is the stability of nuclei on a time scale comparable with the time span needed for a nucleus-nucleus collision. Highenergy collisions ( $E_{\text {lab }}>500 \mathrm{MeV} /$ nucleon) require less than $80 \mathrm{fm} / c$ as far as single-particle properties are concerned. However, it turns out that in order to investigate the fragmentation process in heavy-ion collisions, we have to follow the reaction for a considerably longer time. Unstable fragments are formed which have an excitation energy near the particle-emission threshold, and hence the time for particle emission is quite long, i.e., of the order of a compound nucleus lifetime.

Figure 1 shows how a single nucleon moves in the po-

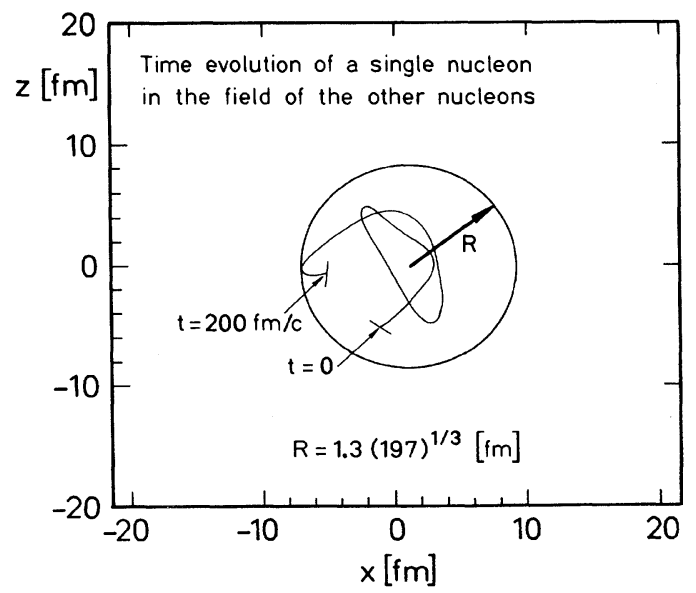

FIG. 1. Trajectory of a single nucleon in the field of 196 others is displayed for a time span of $200 \mathrm{fm} / c$. To visualize the size of the system, we show also a sphere of radius $r=1.3 \times 197^{1 / 3}$. 
tentials generated by all its fellow nucleons in a gold nucleus. For clarity we also show a circle of the radius of $r=1.3 A^{1 / 3}$. One has to keep in mind that in the QMD approach the surface is a consequence of the strength of the mutual interactions and it is not automatically a constant as a function of time. We see that the nucleon moves quite a distance during $200 \mathrm{fm} / c$. Whenever it comes close to the "surface," it is pulled back by the other nucleons. Thus the nucleon remains confined in a sphere.

Figure 2 shows the time evolution of the root-meansquare radius of five nuclei ranging in mass from $\mathrm{Li}$ to $\mathrm{Au}$. For each species the time evolution of the radii of 12 differently initialized nuclei is displayed. For the heavy nuclei we see oscillations around the mean value, but no nucleons are emitted. Light nuclei are a little less stable. One or two of the nuclei emit a nucleon in the time span of $200 \mathrm{fm} / c$ because the local-density approximation is not very good for these light nuclei.

To get rid of unstable configurations, the following procedure can be applied: We select a sample of nuclei which have the required stability; then we choose ran-

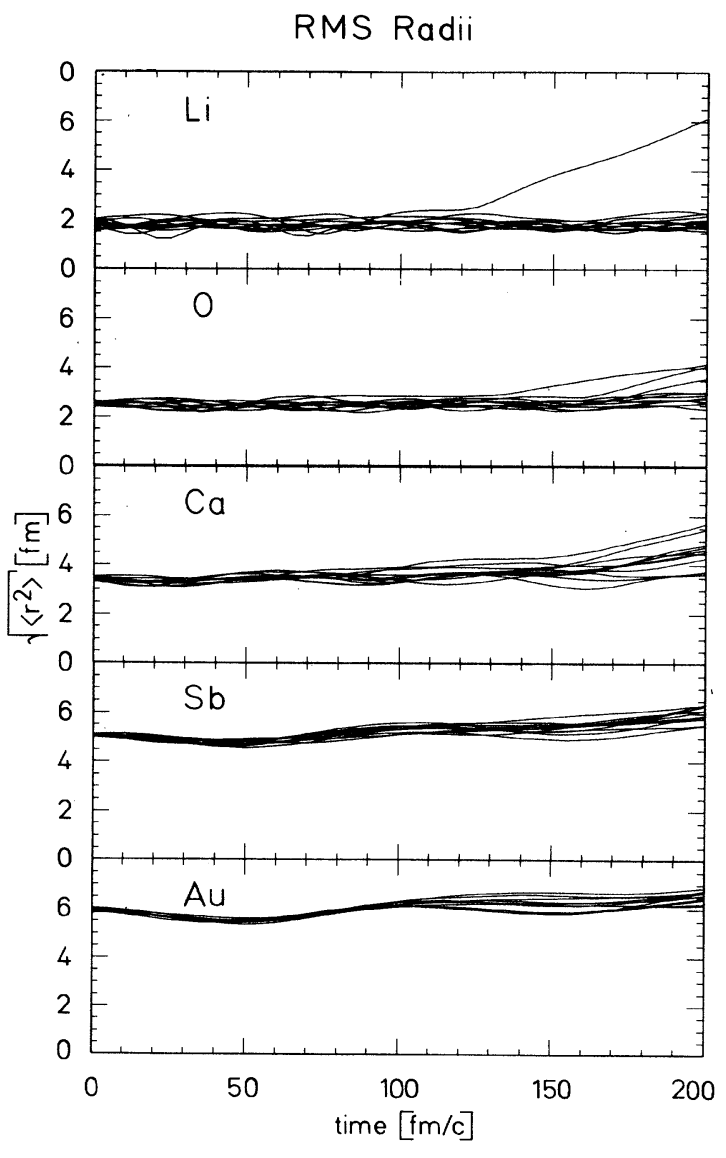

FIG. 2. Root-mean-square radii of different nuclei as a function of time. For each nucleus we display this radius for 12 different initializations. domly two Euler angles and rotate the positions of all nucleons of one nucleus around its center of mass. The rotated nuclei are then boosted toward each other along the old $z$ axis. Each set of Euler angles yields a completely different reaction without changing the stability.

Figure 3 displays the binding energy $\left(\left\langle H_{i}\right\rangle\right)$ per nucleon averaged over all nucleons for the same sample as used in Fig. 2. $H_{i}$ is defined in Eq. (12), and $T_{i}$ is defined as $p_{i 0}^{2} / 2 m$. First of all, we see that the binding energy fluctuates around a mean value. So we have energy conservation on the average. However, the fluctuations reach $2 \mathrm{MeV} /$ nucleon, a large value compared to the average binding energy of $8 \mathrm{MeV} /$ nucleon. In order to appreciate the size of the fluctuations, one has to realize that the potential energy is just the difference between two large quantities, the attractive two-body part $\left(\approx-350 \mathrm{MeV}\right.$ at $\left.\rho=\rho_{0}\right)$ and the repulsive three-body part $\left(\approx 300 \mathrm{MeV}\right.$ at $\left.\rho=\rho_{0}\right)$. Hence a $1-\mathrm{MeV} /$ nucleon fluctuation means that we determine these potentials to an accuracy of 1 part in $10^{3}$. The light nuclei show more fluctuations than the heavier ones. The many nucleons which have to be initialized in the case of heavy nuclei give a longer series of random numbers. This averages

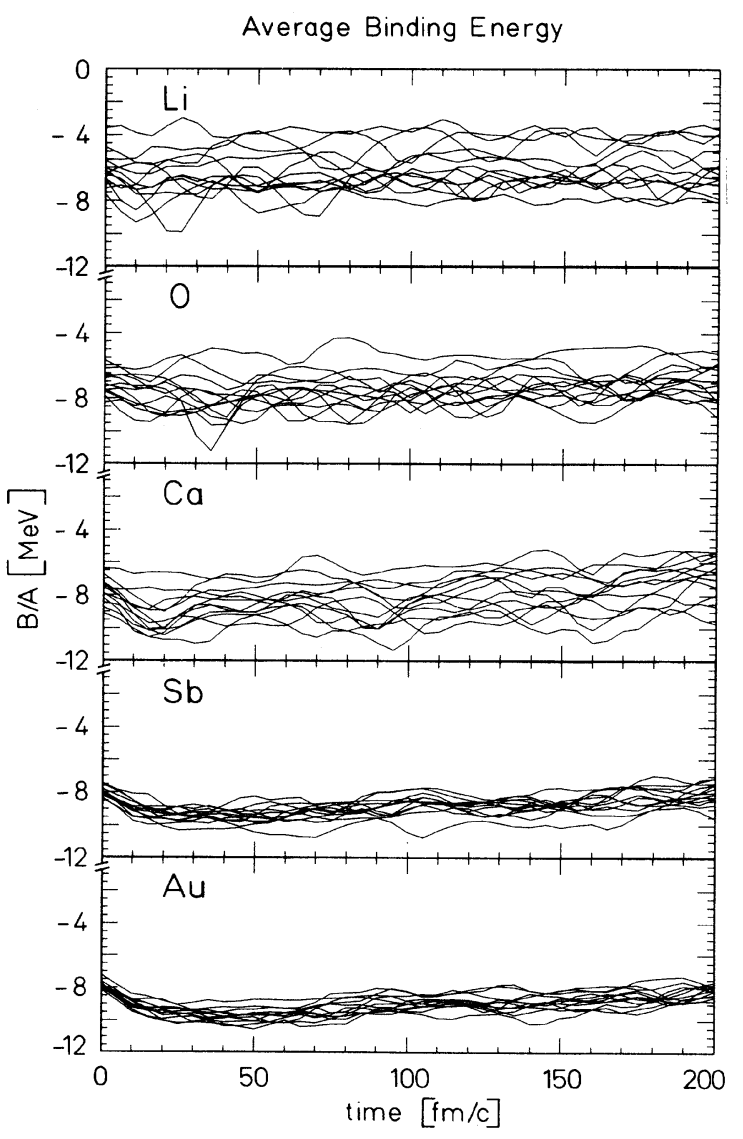

FIG. 3. Binding energy/nucleon as a function of time for the 12 simulations of the different nuclei displayed in Fig. 2. 


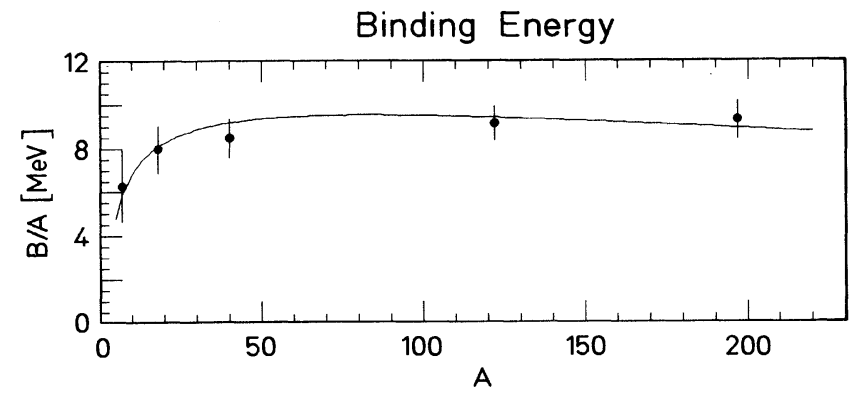

FIG. 4. Average binding energy per nucleon of the nuclei as a function of the mass number $A$. The binding energy is obtained by averaging the binding energy for each individual simulation over the first $100 \mathrm{fm} / c$. Then we average over the 12 simulations. The values are compared with the Weiszäcker mass formula without symmetry energy.

out some of the fluctuations. Thus the energy conservation is here much better than in the BUU calculations, where the use of a grid for the determination of the potential energy makes energy conservation quite difficult.
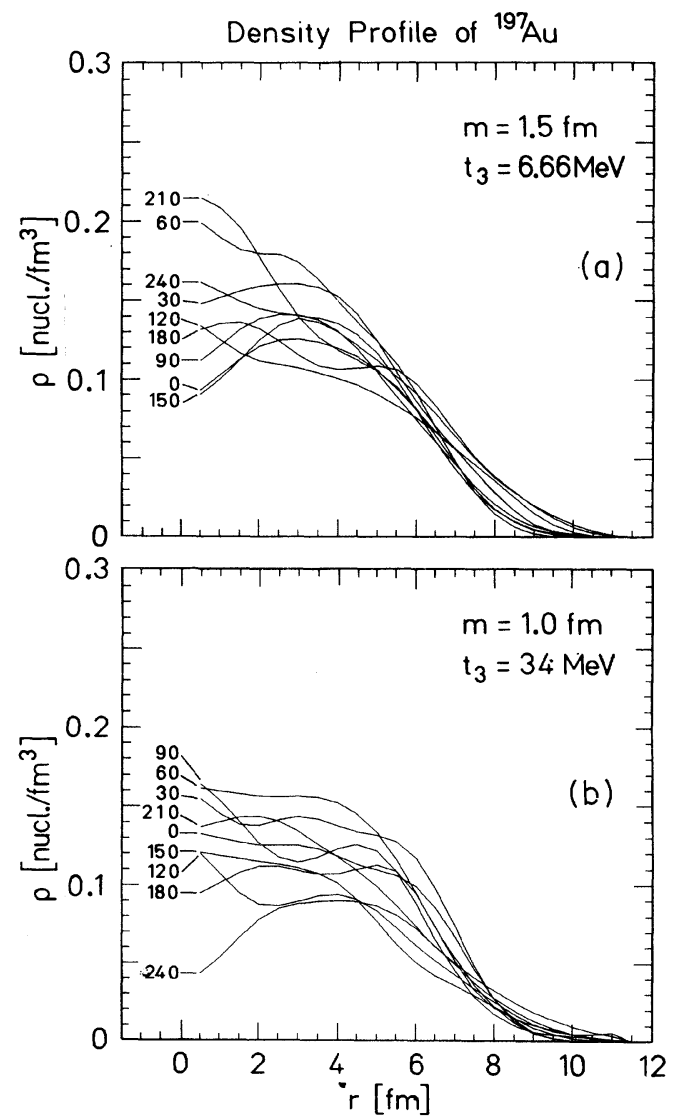

FIG. 5. Radial density distribution for a gold nucleus as a function of time in steps of $30 \mathrm{fm} / c$. (a) shows this distribution for the Yukawa parameters applied in the calculations. (b) shows this distribution for a different choice of these parameters in order to demonstrate the dependence of the surface fluctuation of the Yukawa parameters.
Employing a fourth-order Runge-Kutta method for the time evolution, the energy fluctuations can be substantially reduced; however, the CPU time also increases by a factor of 5 .

The least-bound configurations in the cases of lithium and oxygen are those which emit particles the earliest (see Fig. 2). Discarding these initial configurations, which have a low binding energy, allows a further reduction in the number of unstable nuclei. This cut can be applied when it is important to keep light nuclei stable for a long time.

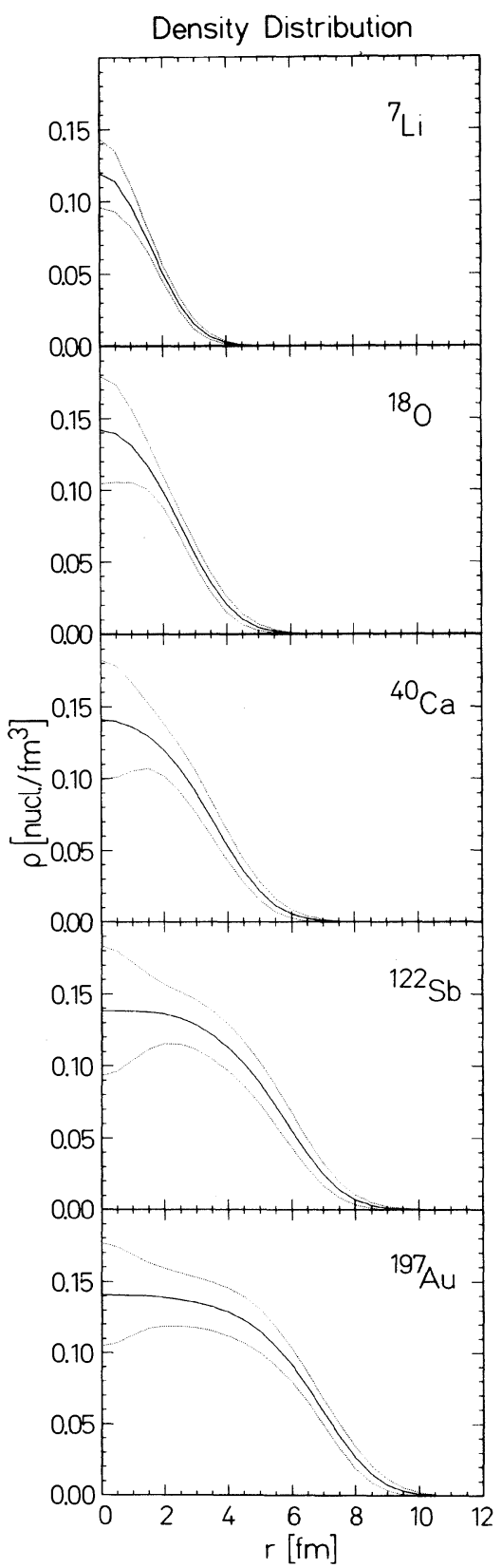

FIG. 6. Radial density distribution of the nuclei. We have averaged the local density over the first $100 \mathrm{fm} / c$. The mean value and as well, the standard deviation (dotted lines) are plotted. 
Figure 4 displays the average binding energy per nucleon of the nuclei. These numbers are average values over the first $100 \mathrm{fm} / c$ and over the 12 simulations. For low masses, not only the trend, but also the absolute values are reproduced. At large masses the binding energy becomes constant and the heavy nuclei are overbound by $1 \mathrm{MeV} /$ nucleon. We do not see a maximum in the binding energy in the region of iron as we did not include the symmetrization energy.

More important than the reproduction of the rootmean-square radius is the requirement that the nucleus keeps its radial distribution. In Fig. 5 the radial distribution of a gold nucleus is shown for two different parameters of the Yukawa potential. We display the density profiles in time steps for $30 \mathrm{fm} / c$. In Fig. 5(a) we see the distribution with the Yukawa parameters chosen in our calculations. We observe that the nuclear surface is preserved for almost $240 \mathrm{fm} / c$. To understand the large fluctuations in the interior, one has to recall that there are very few (about four) nucleons. We will see that these fluctuations average out as a function of time. In Fig. 5(b), with different Yukawa parameters, the nuclear surface is much less well preserved, although the rms radius as well as the nuclear binding energy are very close to those of Fig. 5(a).

If we average the density over the 12 nuclei and over the first $100 \mathrm{fm} / c$, we find a quite smooth density distribution. It is displayed in Fig. 6. Our surface thickness is slightly too large as compared to that extracted from electron-scattering experiments; however, the overall features are quite nicely reproduced. Because of the few nucleons present, we are not able to avoid fluctuations of the central density. In order to make the density profile as accurate as possible, we take care that, between 15 and $40 \mathrm{fm} / c$ when colliding nuclei reach their maximal density and the transverse momentum is built up, the central density of a single nucleus agrees with the values obtained in Hartree-Fock calculations [45]. They yield a central density of 0.155 nucleon $/ \mathrm{fm}^{3}$ for static nuclei. These densities are around $10 \%$ lower than those obtained in BUU simulations. Probably this explains the fact that in nucleus-nucleus collisions BUU calculations yield a higher maximal central density than QMD calculations.

\section{REACTION $84 \mathrm{MeV} / \mathrm{NUCLEON}{ }^{18} \mathrm{O}+{ }^{197} \mathrm{Au}$}

\section{A. Survey of the reaction}

\section{Final momenta of protons and fragments}

To investigate the nuclear fragmentation in the intermediate-energy regime, we performed 1700 simulations between the impact parameters $b=0$ and $13 \mathrm{fm}$. The impact parameter for each simulation was drawn randomly from a distribution $F(b)=b^{2}$. Thus the sum over all simulations can directly be compared with inclusive data. As mentioned in Sec. II, we used a static soft equation of state [35] and an isotropic cross section of $40 \mathrm{mb}$ [35], which yield about the same result as the cross section calculated from the $G$ matrix [44]. In Fig. 7

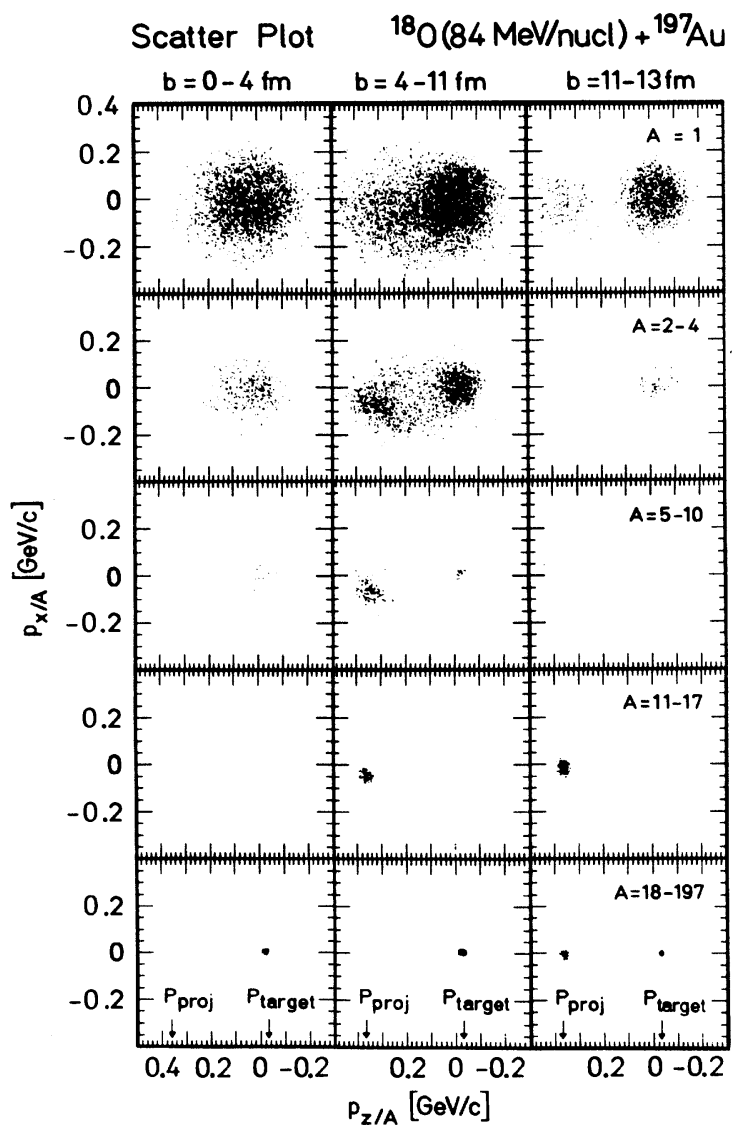

FIG. 7. Final momenta of the particles in the reaction plane. We display the final longitudinal momentum $p_{z} / A$ and transverse momentum $p_{x} / A$ in the reaction plane for all nucleons and clusters, separated into three impact-parameter bins and five different classes of nucleons and clusters.

we display the final c.m. momentum per nucleon of all nucleons and fragments in the reaction plane. ( $z$ is the beam direction and $x$ the direction of the impact parameter.) We separate the simulations into three impact parameter bins and five fragment classes. In the lowest impact-parameter bin, we have a complete overlap of projectile and target. In the next bin the overlap is only partial. The last bin shows typical peripheral reactions. The mean values of the momenta for the different bins and the different types of fragments are collected in Table I.

Initially, the gold and oxygen nuclei have longitudinal momenta per nucleon of -33 and $364 \mathrm{MeV} / c$, respectively. Before the nuclear interaction starts, they have gained transverse momentum and have slowed down a little as a result of the Coulomb interaction.

For small impact parameters and light particles, we observe an almost isotropic emission. The velocity of the emitting source, however, is not that expected for a compound nucleus $\left(\left\langle p_{z}\right\rangle=0\right)$ but larger. Hence there contribute many projectilelike nucleons which emerge from the combined system before they have been slowed down 
TABLE I. Final c.m. momenta in the reaction plane $\left(p_{x}, p_{z}\right)$ for different impact parameters $b$ and different fragment masses $A$.

\begin{tabular}{cccc}
\hline \hline $\begin{array}{c}b \\
(\mathrm{fm})\end{array}$ & $\boldsymbol{A}$ & $\begin{array}{c}\left\langle p_{z} / A\right\rangle \\
(\mathrm{MeV} / c)\end{array}$ & $\begin{array}{c}\left\langle p_{x} / A\right\rangle \\
(\mathrm{MeV} / c)\end{array}$ \\
\hline$<4$ & 1 & 53 & -12 \\
& $2-4$ & 63 & -12 \\
& $5-10$ & 24 & -5 \\
& $11-17$ & -16 & -15 \\
& $>18$ & -21 & 4 \\
$4-11$ & 1 & 75 & -29 \\
& $2-4$ & 122 & -37 \\
& $5-10$ & 227 & -55 \\
& $11-17$ & 340 & -47 \\
& $>18$ & -25 & 6 \\
& 11 & 35 & -5 \\
& $2-4$ & 14 & -6 \\
& $5-10$ & 94 & -15 \\
& $11-17$ & 360 & -13 \\
& $>18$ & -30 & -1 \\
\hline \hline
\end{tabular}

completely. The momentum transfer is not complete, even at the most central collisions. Larger fragments in central collisions come more dominantly from the target remnant. Their average velocity is smaller than that of protons, but larger than that of the heavy remnant. Again, the distribution is almost isotropic with about a $10 \%$ enhancement in forward direction. There are very few fragments in the mass range 11-17, but we observe in each simulation one heavy remnant, which by the emission of some tens of nucleons becomes cold and consequently stable. From the average momentum of these remnants, we see that the momentum transfer is by far not complete. The projectile does not survive a central collision as an entity. In QMD calculations the central collisions are the main source of IMF production, in agreement with recent experimental findings [24].

The next impact-parameter bin reveals two sources for light particles $(A<5)$ and, even more pronounced, for light fragments. Both the projectile and target source have slowed down, and we observe negative-angle $\left(p_{x}<0\right)$ scattering of the projectile remnant. Initially, the projectile has $p_{x}>0$ as a result of Coulomb repulsion. This observation is exactly opposite to the behavior at larger energies where, because of the compressional energy, the net transverse momentum transfer of the nuclear interaction is parallel to that caused by the Coulomb force. At lower energies than investigated here, these intermediate impact parameters lead to deep-inelastic collisions [4,5]. In almost all cases there is a projectile remnant and always a target remnant. The size of the projectile remnant depends strongly on the impact parameter. Few targetlike IMF's are produced at these impact parameters.

In the peripheral reactions projectile and target keep their identity. They exchange little momentum and gain little excitation energy, which causes the emission of few nucleons and seldomly of light fragments.

\section{Can the impact parameter be measured?}

The impact parameter of a heavy-ion collision cannot directly be measured, of course. But we can ask the question: Are there observables which allow one to determine the impact parameter indirectly? At much higher energies it has been found that there are two observables which are strongly correlated with the impact parameter: (i) The total multiplicity of protons and (ii) the mass number of the heaviest fragment.

Figures 8 and 9 show the total multiplicity and mass number of the heaviest fragment as a function of the impact parameter for the investigated reaction. For each proton multiplicity $N_{p}$ and target remnant $A_{\max }$ we calculate the average impact parameter and its dispersion. We see that for $b>4 \mathrm{fm}$, i.e., for not completely overlapping reactions, both observables allow a very accurate determination of the impact parameter. Note that in QMD calculations no fission occurs. Fission events are expected at large impact parameters and yield two fragments with roughly half of the target mass. Similar to higher energies, the total multiplicity becomes independent of the impact parameter as soon as projectile and target overlap completely. This is due to a very similar energy deposit and momentum transfer in these central events.

\section{B. Observables}

\section{Double-differential proton cross section}

The double-differential proton spectra for this reaction have been measured by Brummund [16]. We compare our calculation with their results in Fig. 10. The lines are the data, and the circles are the result of our calculation. The error bars contain the statistical error only. The slopes at the different angles are well reproduced. This is remarkable since, as we have seen, they are generated by summing over particles of quite different origin. In the

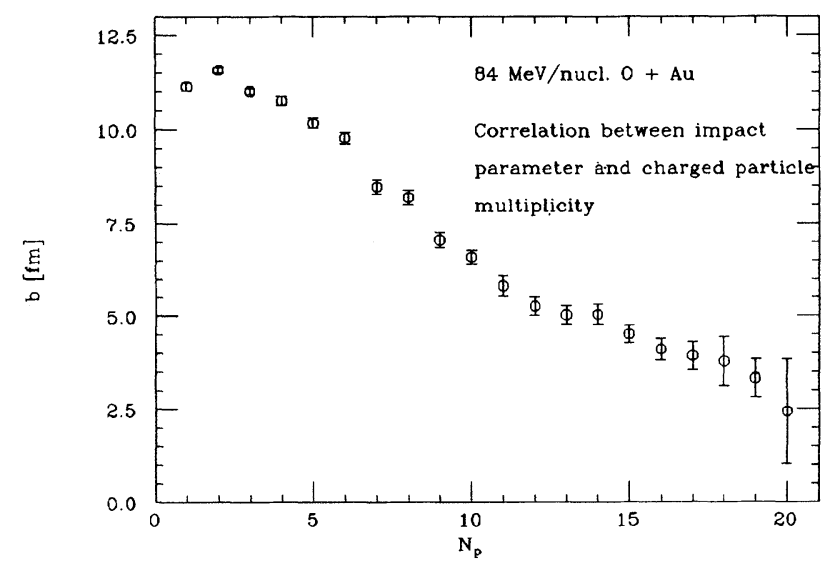

FIG. 8. Correlation between the proton multiplicity $\boldsymbol{M}_{p}$ and impact parameter $b$. 


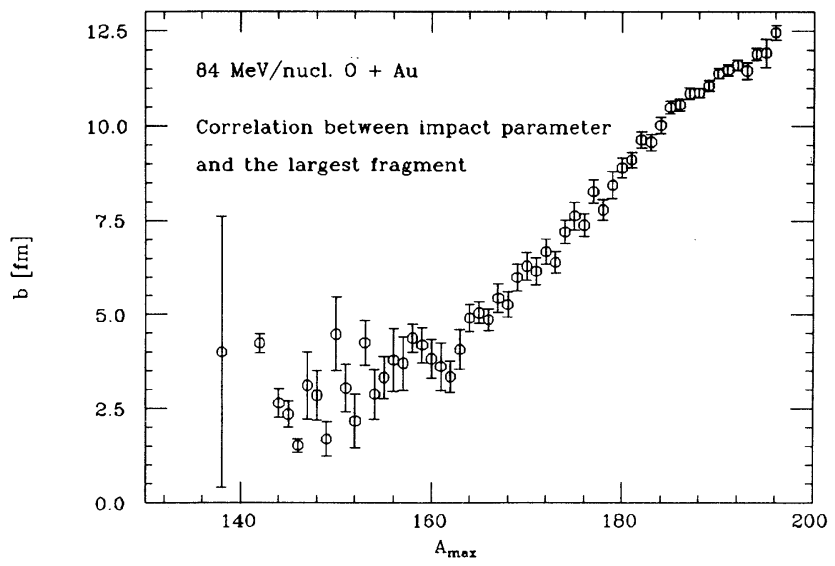

FIG. 9. Correlation between the heaviest fragment (target remnant) and impact parameter $b$.

forward direction we have the largest contribution from emission from projectilelike remnants. At larger angles we are more sensitive to the source (around midrapidity) generated in central collisions. At the backward angles we have mainly nucleons emitted very late from the target remnant. The reproduction of the absolute yield is less satisfactory. However, one should note also that experimentally the determination of the absolute value of the cross section seems to be difficult. For a very similar reaction $84 \mathrm{MeV} /$ nucleon ${ }^{12} \mathrm{C}+\mathrm{Au}$, which has in addition been measured by the Lund group, both experimental data sets differ by a factor of 2 for the absolute value of the cross section [4,5]. We would like to stress once more that the inclusive proton spectrum is incompatible with the assumption that they originate from an isotropically emitting source.

The proton spectrum would be anisotropic also in any system which is displaced by a fixed vector $\mathbf{p}$ in momentum space and thus shows that there is nothing like a single isotropically emitting source in this reaction. This can be easily seen in Fig. 7 .

\section{Mass-yield curve}

Figure 11 displays the total mass yield observed in our calculation for masses larger than 100 . We observe an exponential decay as a function of $A-A_{\text {target }}$. There exist no fragments in the mass range $26 \leq A \leq 100$. Please note that the QMD calculation cannot reproduce fission. From this result we can conclude that the limited excitation energy does not allow the production of remnants which fall into this mass range. There are no data available for this reaction. Aleklett et al. [21], however, have measured the mass yield of those heavy remnants, which can be detected by radiochemical methods, for the very similar reaction $84 \mathrm{MeV} /$ nucleon $\mathrm{C}+\mathrm{Au}$. Thus we compare our results with their data. If one takes for granted that the radioactive remnants are typical for all remnants, we observe a quite good agreement down to a mass loss of 40 units. Below that our statistics break down. This means that the average emission chains of excited QMD nuclei are quite realistic as far as the average multiplicity of emitted particles is concerned.

The form of the distribution on the low-mass side is dominated by the projectilelike fragments. The targetlike

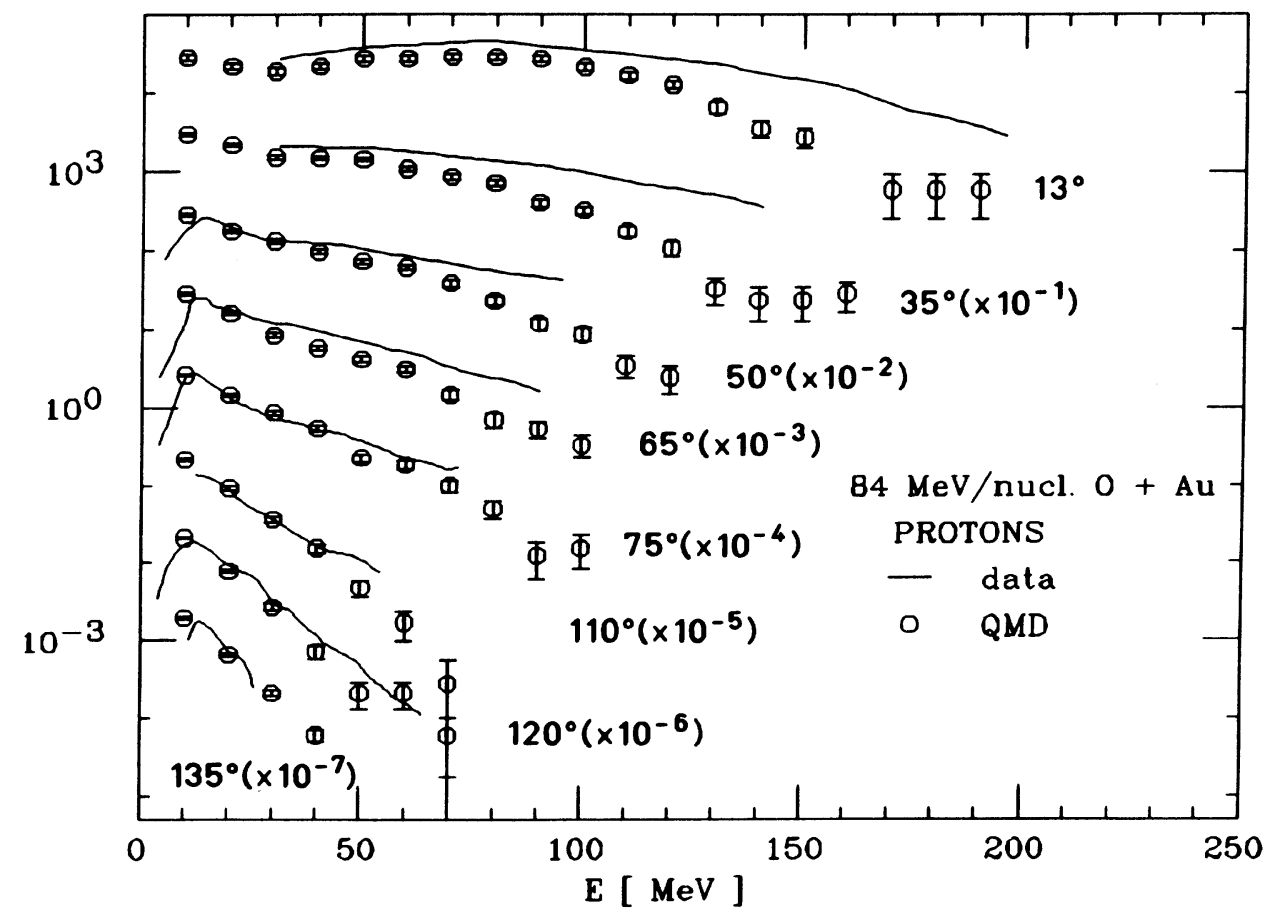

FIG. 10. Double-differential cross section $d \sigma / d \Omega d E$ for protons in the reaction $84 \mathrm{MeV} /$ nucleon ${ }^{18} \mathrm{O}+\mathrm{Au}$. The QMD calculations are compared with the data of Brummund et al. [16]. 


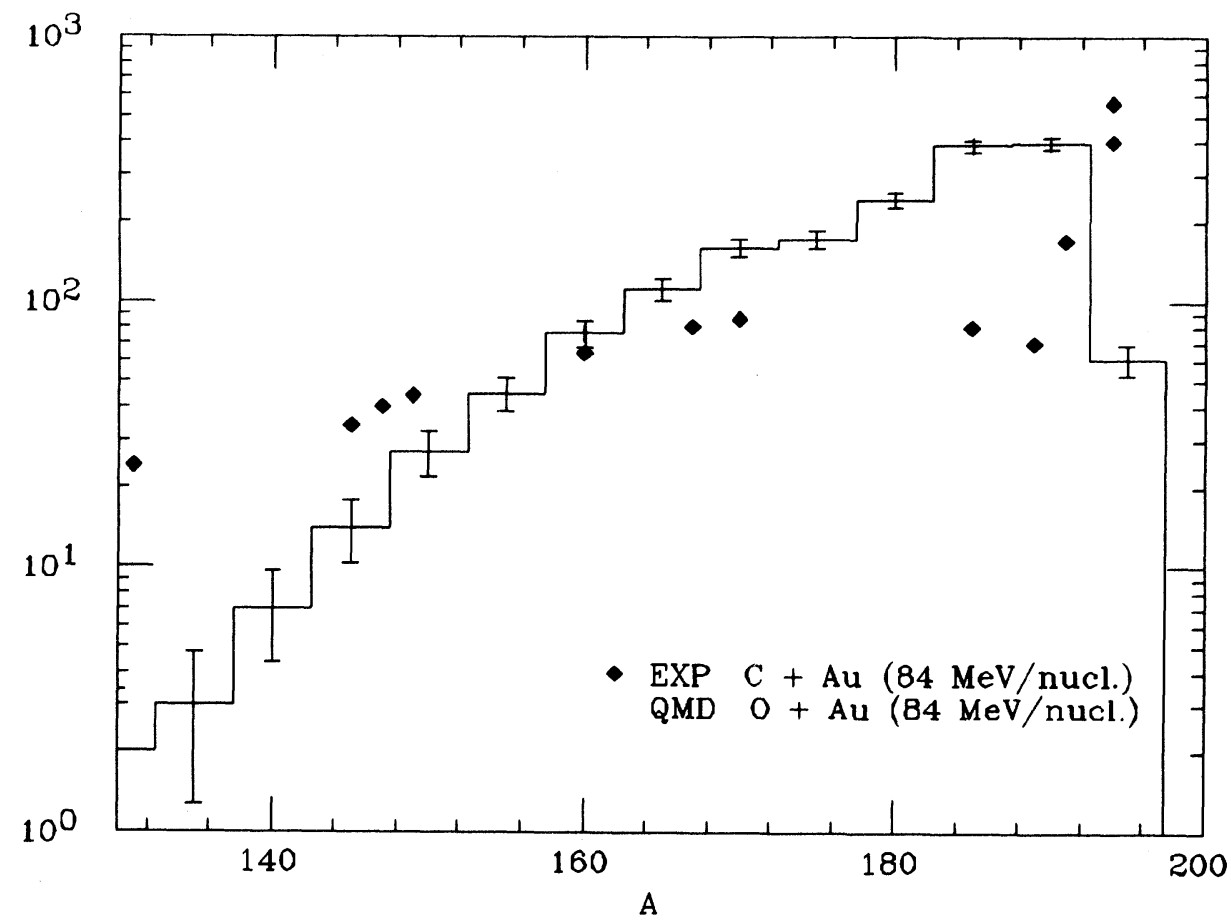

FIG. 11. Impact-parameter-averaged mass yield of the heavy remnant as compared to the data of Aleklett et al. [21].

medium-mass fragments show the usual power-law distribution $\sigma(Z) \sim Z^{-\tau}$, as can be seen from Fig. 12. The exponent $\tau$ is compatible with the experimental result $\tau \approx 2.3[10,17]$. Thus, also, at this low energy we observe this common form of the charge-yield curve which has been observed for virtually all nuclear fragmentation processes [31]. This is also a strong hint that we are at this energy at the low-energy side of typical multifragmentation processes and not at the upper limit of compound evaporation.

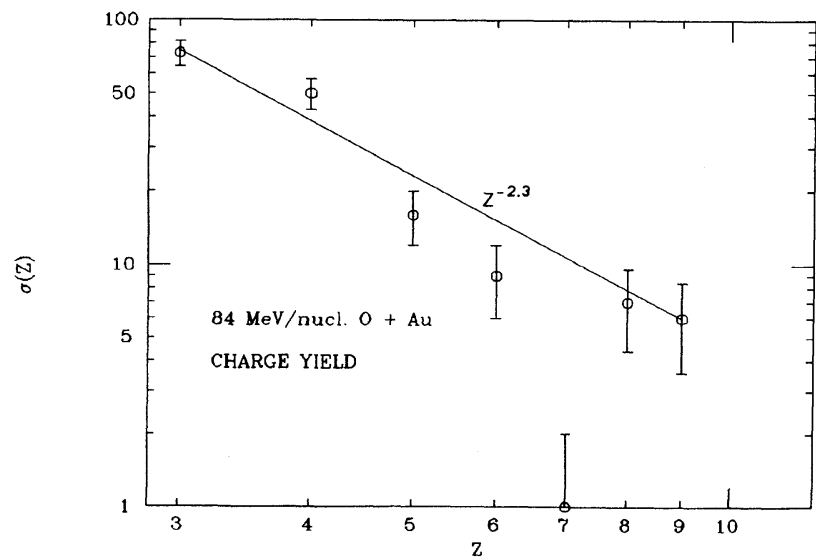

FIG. 12. Charge yield of targetlike fragments as compared to experiment. The experimental curve can be fitted by a power law $\sigma(Z)=Z^{2.3}$.

\section{Associated proton multiplicity}

The average proton multiplicity $\left\langle M_{p}\right\rangle$ of events in which a fragment of mass $A$ is produced is displayed in Fig. 13. The upper curve shows the multiplicity in $4 \pi$, and the lower curve corresponds to the experimental cuts $23^{\circ}<\vartheta_{\text {lab }}<78^{\circ}$. As expected, from Figs. 8 and 9 we see a strong dependence of $\left\langle M_{p}\right\rangle$ on the mass number of the heavy residue. It is interesting to note that at the same

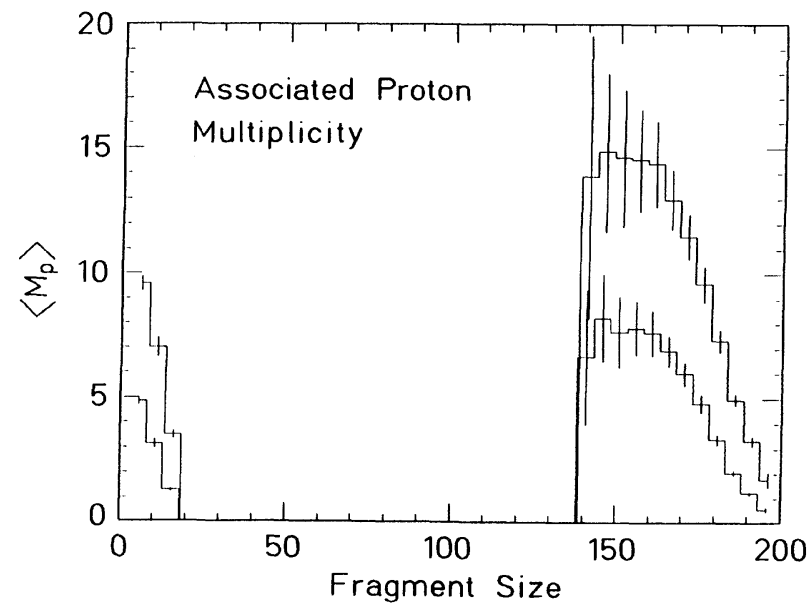

FIG. 13. Proton multiplicity $M_{p}$ as a function of the fragment size. We display the total multiplicity (upper line) and the multiplicity observed with the experimental setup (lower line). 
impact parameter the associate proton multiplicity is lower in those events, in which an IMF is produced as compared to those without an IMF. It costs energy to produce an IMF, and hence the excitation energy left is lower if such a fragment is produced. Consequently, the number of emitted protons is smaller.

\section{Momentum transfer - of use as a signal for equilibration?}

One of the most interesting questions in this intermediate energy regime is whether in central asymmetric heavy-ion collisions the system reaches global equilibrium. In events where projectile and target overlap only partially, we do not expect equilibration. In inclusive experiments these peripheral events contribute to the measured spectra, and hence one has to disentangle the contribution from central and peripheral events. There are two methods which were applied frequently. Either one concentrates on phase-space regions $\left(\vartheta_{\text {c. m. }} \approx 90^{\circ}\right)$, where just from geometrical considerations the contribution from peripheral collisions may be small. We will come back to this procedure later. Or one assumes that the spectra can be described by a multitude of isotropically emitting sources with different source velocities. The last method suffers from the open question whether these sources can indeed be identified or whether the whole procedure is nothing but a multiparameter fit of the data which does not allow to infer on equilibrium.

One of the observables which allow the investigation of this question in detail is the momentum transfer to the heavy residue. The velocity of the remnant should be equivalent to the source velocity of those nucleons which are emitted from the target-remnant source because the emission of nucleons does not change this velocity on the average.

In Fig. 14 we display the final longitudinal and transverse momentum of the residues as a function of the impact parameter. We see, first of all, that even in the most central collisions the collective momentum transfer $\Delta p=\left(p_{\text {initial }}-p_{\text {final }}\right) / p_{\text {initial }}$ is only $40 \%$ and decreases with increasing impact parameter. We observe also a considerable transverse momentum transfer caused by the attraction of the nuclear interaction. The transfer corresponds to negative angle scattering. Projectile and target form a combined system and rotate around each other for a short period of time.

Unfortunately, the remnant velocities have not been measured for the reaction ${ }^{18} \mathrm{O}+\mathrm{Au}$, but they have been measured in the very similar reaction $\mathrm{C}+\mathrm{Au}$ at the same energy with radiochemical methods [21]. In Fig. 15 we compare this experiment with theory. First of all, we observe that the measured energies are very low (in the region of some $\mathrm{keV} /$ nucleon). Even remnants that have lost 60 nucleons and hence have to come from central collisions have an energy of little more than 0.3 $\mathrm{MeV} /$ nucleon. This corresponds to a velocity of $0.018 c$. The calculation agrees quite nicely with the experimental data in the trend as well as in the absolute value. The error bars mark the fluctuations,

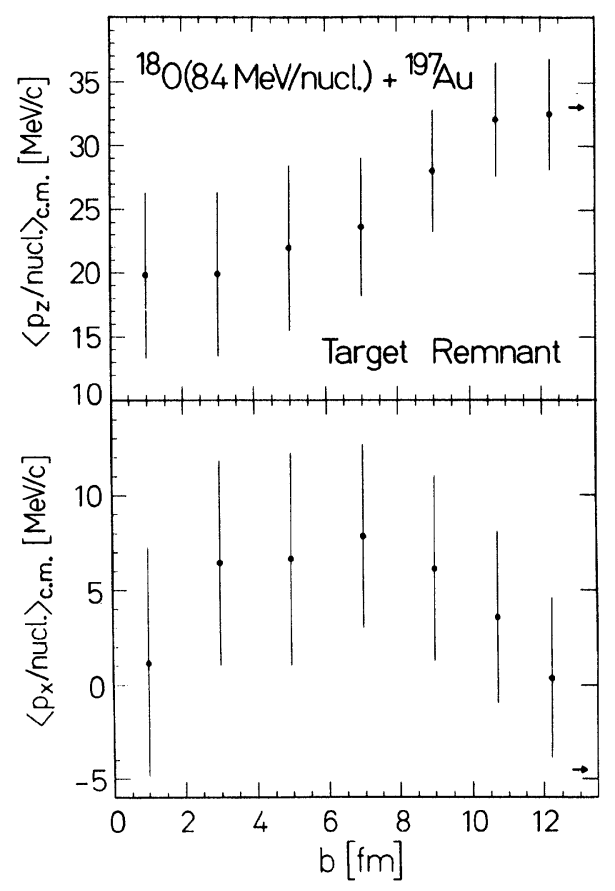

FIG. 14. Final longitudinal momentum $p_{z / A}$ and transverse momentum $p_{x} / A$ in the reaction plane for the target remnant as a function of the impact parameter.

$$
\Delta=\left[\frac{1}{N} \sum_{i}^{N}\left(E_{i}-\bar{E}\right)^{2}\right]^{1 / 2},
$$

in the energy distribution observed for fragments of the same mass. Here $E_{i}$ is the kinetic energy per nucleon of fragments with a given mass number $A$ and $N$ is the number of events in which these fragments have been observed. These very low momenta of the target residues have to be confronted with the rather high average mo-

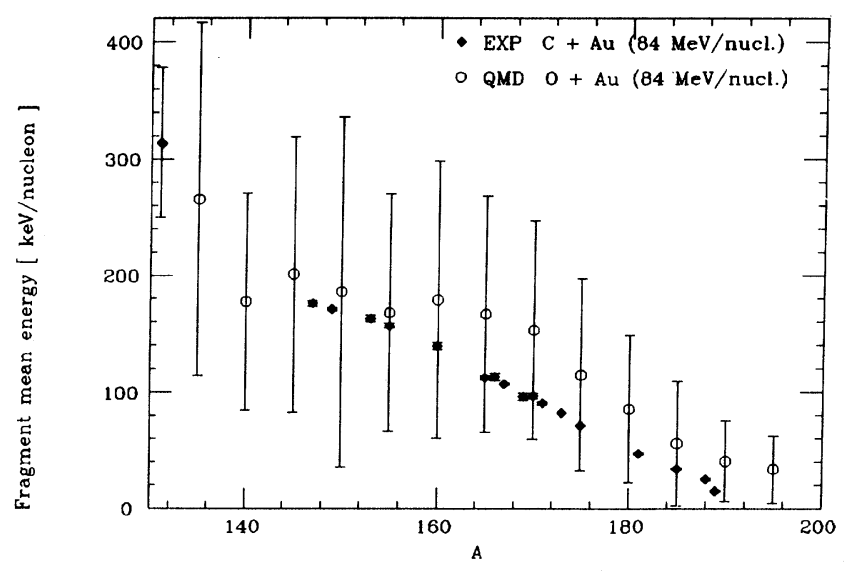

FIG. 15. Final kinetic energy per nucleon. We compare the experimental results for the reaction $84 \mathrm{MeV} /$ nucleon $\mathrm{C}+\mathrm{Au}$ [21] with our calculation for the reaction $84 \mathrm{MeV} /$ nucleon ${ }^{18} \mathrm{O}+\mathrm{Au}$. 
menta of the targetlike IMF's. The experimental momentum distribution of IMF's has been parametrized by a two-source fit [18]. However, emission of the fragments of mass 6-16 is dominated by one source, called the "intermediate source." The extracted source velocities which - in a thermal model-should correspond to the average velocity of the remnant is in between $0.07 c$ and $0.049 c$. These values agree with our calculation. Experimentally, it has been observed that the charge of the particles with $Z<15$ can only account for at most $50 \%$ of the total charge of the system. Therefore, always one heavy remnant is left. If the IMF's originate from an equilibrated system, there must be a class of target remnants which has the same average velocity as the IMF's. As we have seen, experiment shows that the average velocity of the IMF's is always much larger. Hence, combining all the experimental data, one can rigorously exclude an equilibrated system as the source of the IMF's.

Because of the widespread use of thermal models in this energy domain, it is certainly worthwhile to discuss some of the approaches and to compare their assumption with our calculation.

A while ago it was argued [46,47] that from isotopic ratios measured in inclusive experiments the "entropy" of the system can be extracted. To investigate this question in detail, Hahn and Stöcker subsequently advanced a quantum statistical mode [34]. This model assumes that the system thermalizes in the course of the reaction and can then be described completely by the density and temperature. It assumes that the decay of the thermalized system is determined by phase space only, i.e., that all final states of nucleons and fragments (including particle unstable final states) are equally probable. It turns out that the fragment yield can then be directly related to the entropy of the system.

This approach is only meaningful if such a thermalized system can be identified even in inclusive data. It was conjectured that restricting the analysis to light particles emitted under $\vartheta=90^{\circ}$ in the nucleus-nucleus c.m. system, i.e., to those particles which underwent hard scattering, one would be sensitive to central collisions, where indeed the system comes closest to equilibrium.

Our calculation shows that this hope is in vain. In Fig. 16 we display an impact-parameter distribution of protons observed under $\vartheta_{\text {c.m. }}=90^{\circ}\left( \pm 10^{\circ}\right)$. We see that all impact parameters contribute to the spectrum at this angle. For impact parameters up to $6 \mathrm{fm}$, the contribution to the spectrum is proportional to $b$. Thus the emission probability is constant. At larger impact parameters, the probability becomes lower. Then the angular distribution of the protons becomes increasingly anisotropic, showing peaks around $\vartheta_{\text {c.m. }}=0^{\circ}\left( \pm 10^{\circ}\right)$ and $\vartheta_{\text {c.m. }}=180^{\circ}\left( \pm 10^{\circ}\right)$ (compare Fig. 7). Thus, in inclusive data even at $\vartheta_{\text {c.m. }}=90^{\circ}$, we see particles from different reaction types and the assumption that all come from one thermalized source cannot be substantiated. Hence an entropy in the thermodynamical sense (as a measure for the number of accessible states) cannot be derived from such inclusive data. However, the situation improves if yield ratios of IMF's rather than protons are studied at $\vartheta_{\text {c.m. }}=90^{\circ}$. As we can see in Fig. 7 , the IMF's with $11 \leq A \leq 17$ come

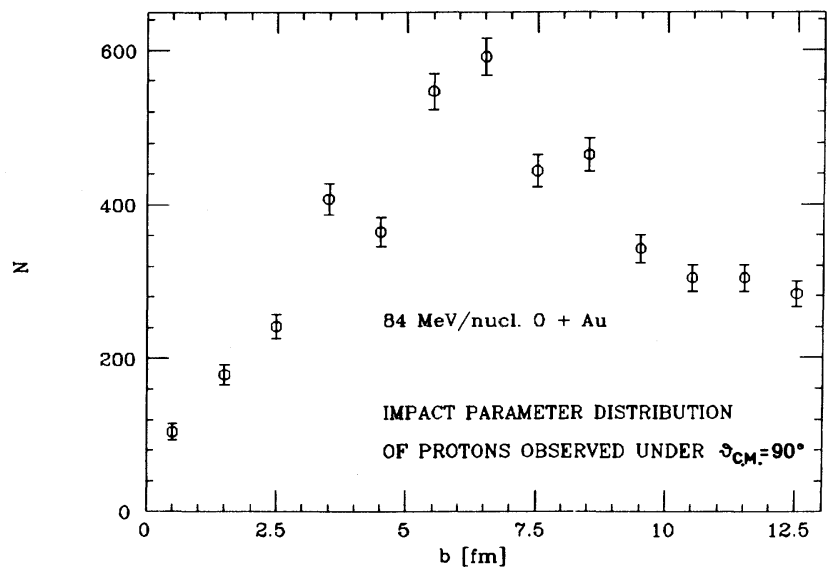

FIG. 16. Origin of the protons observed under $\vartheta=90^{\circ}$ in the c.m. system. We display the number of particles which contribute to the spectrum at $\vartheta_{\text {c. m. }}=90^{\circ}$ as a function of the impact parameter $b$.

predominantly from central collisions. It has been shown at higher energies, where multiplicity-selected data have been provided by the plastic ball group, that for inclusive experiments the numerical value of the entropy (measured by the simple formula $S / A=3.95-\ln R_{d p}$, where $R_{d p}$ is the deuteron to proton ratio) has nothing to do with the true thermodynamical entropy of the system. This is due to the observation that an impact-parameteraveraged deuteron-to-proton ratio yields quite different results for $\langle S / A\rangle$ as compared to the averaging of $S / A$ for different impact parameters. Here it is assumed that the total multiplicity provides a good measure for the impact parameter. The numerical values for the entropy for high-multiplicity-selected data are much smaller than that deduced from inclusive data [48]. However, even at this higher energy equilibrium is not established, and, hence the physical meaning of the number-dubbed entropy remains unclear.

The different range of impact parameters which contribute to the spectra may be also the reason why the entropy determined from the proton-to-tritium ratio is larger than that determined from heavy fragments [11] for the reaction $\mathrm{C}+\mathrm{Au}$ at $84 \mathrm{MeV} /$ nucleon.

\section{Memory of the reaction plane}

Another quantity which allows one to investigate the degree of thermalization is the probability of out-of-plane emission. Almost all statistical models applied to heavyion reactions assume that the angular momentum of the combined system can be neglected, and therefore the emission is isotropic in the azimuthal angle $\phi$. In Fig. 17 we show the out-of-plane correlation predicted by the QMD model. We display

$$
R=\frac{\left\langle p_{x}^{2}\right\rangle-\left\langle p_{y}^{2}\right\rangle}{\left\langle p_{x}^{2}\right\rangle+\left\langle p_{y}^{2}\right\rangle},
$$




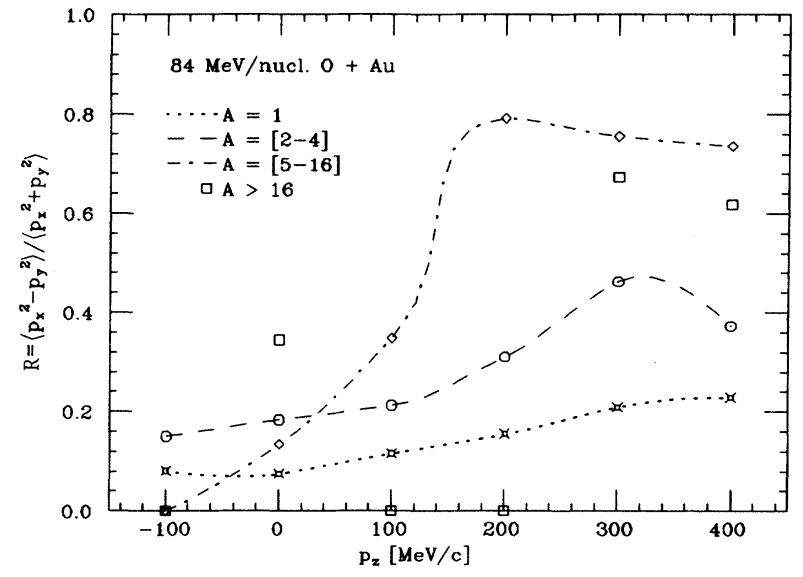

FIG. 17. Out-of-plane correlation $R=\left(\left\langle p_{x}^{2}\right\rangle-\left\langle p_{y}^{2}\right\rangle\right) /$ $\left(\left\langle p_{x}^{2}\right\rangle+\left\langle p_{y}^{2}\right\rangle\right)$ as a function of the final transverse momentum in the c.m. system for different fragment classes.

as a function of the longitudinal momentum. For isotropic emission this quantity is zero; a positive value indicates a preferential emission in the reaction plane. We see that the anisotropy increases with the fragment size, but even protons show a remarkable in plane enhancement. This enhancement increases with increasing longitudinal momentum. Targetlike light fragments have $R=0.2$, which corresponds to $\left\langle p_{x}^{2}\right\rangle /\left\langle p_{y}^{2}\right\rangle=1.5$. Even more aligned are the projectile remnants, which can hence be used to determine the reaction plane. Thus the out-of-plane correlation of protons and fragments would be a good tool to distinguish between these isotropically emitting thermal models and dynamical models such as QMD.

It should be noted that at higher energies, $E_{\text {lab }} \geq 150$ $\mathrm{MeV} /$ nucleon, this in-plane correlation has been measured for a variety of projectile-target combinations by the Plastic Ball Collaboration [49]. They also observe the reverse phenomenon: The stopped particles at midrapidity are preferentially emitted perpendicular to the reaction plane. This feature is not seen at this low energy.

\section{Multiplicity of IMF's}

The next figure, Fig. 18, shows the multiplicity in $4 \pi$ of targetlike IMF's as compared to the experimental data which show the relative probability to detect a fragment in coincidence with a fragment of $Z \geq 6$. The straight line shows the extrapolation of the measured data to $4 \pi$ [10]. For reasons of low statistics we ar not able to trigger on $Z \geq 6$ fragments in the calculation, but use all events. This is reasonable because it has been observed experimentally that the average multiplicity only increases mildly with the charge of the trigger fragment [18]. Thus the experimental multiplicity distribution averaged over all events should be slightly steeper than that for $Z \geq 6$ trigger particles and thus be even, in agreement with the calculations. This allows the conclusion that the basic mechanism for fragmentation is well de-

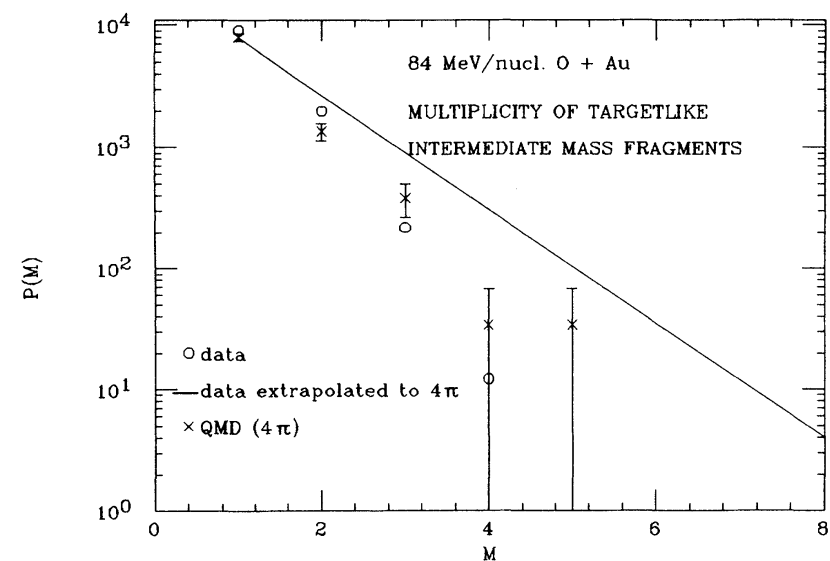

FIG. 18. Multiplicity of targetlike IMF's as compared to the experimental results.

scribed in the QMD approach, even at this low beam energy.

\section{Angular distribution of IMF's}

The angular distribution of the IMF's is displayed in Fig. 19. We show separately the positive- and negativeangle scattering as obtained from the calculations. Experimentally, this distinction is only possible if one measures the direction of the target angular momentum by the polarization of emitted $\gamma$ rays. Otherwise, one has to identify $+\vartheta_{\text {c.m. }}$ and $-\vartheta_{\text {c.m. }}$. We start the discussion with the distribution of fragments with mass number $A=18$. They are observed close to $0^{\circ}$, but the negative angles are slightly preferred. This means that (despite the Coulomb force, which leads to positive angles) close to the surface the nuclear interaction is sufficiently attractive to overcome the repulsive Coulomb force and to pull projectilelike fragments toward negative scattering angles. This effect increases with decreasing mass of the projectile remnant, i.e., with the centrality of the reaction. Because

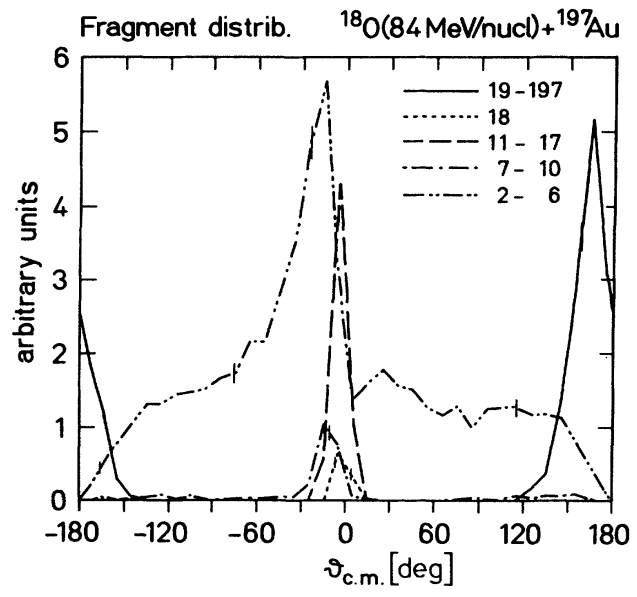

FIG. 19. Angular distribution of the fragments. 
of momentum conservation, the target remnant shows just the opposite feature; i.e., it appears at positive angles, although the Coulomb force alone would have driven it to negative angles. Intermediate-mass target fragments show a fairly isotropic distribution.

\section{Correlations between protons and projectilelike fragments}

It has been reported [18] that protons measured in coincidence with an IMF in forward direction are predominantly observed under $\Delta \phi=\phi_{\text {frag }}-\phi_{p} \approx 180^{\circ}$. This correlation increases with increasing proton energy. Combining these results with measurements of the $\gamma$ polarization [50] in coincidence with protons, one can draw the conclusion that the protons are preferably emitted under negative angles, whereas the fragment is observed under positive angles. We investigate this correlation also in our approach. Figure 20 displays the final momenta of fragments (circles) and coincident protons (dots). We have applied here the experimental cuts $3^{\circ}, \vartheta_{\text {frag }}<11^{\circ}$ and $23^{\circ}<\vartheta_{p}<78^{\circ}$. We do not find the experimental correlations, but see exactly the opposite. With increasing proton energy the relative azimuthal angle between fragment and proton is peaked around zero. This is due to the fact that the fragments are on the "wrong side" in the QMD calculations. Most of those coincidence events come from more peripheral collisions $(b>8 \mathrm{fm})$. It seems that the nuclear force used in the QMD calculations is too attractive close to the surface.

\section{Fragment-fragment correlations}

Fragment-fragment correlations have recently received a lot of interest because it was conjectured that the distribution of relative velocities between two IMF's emitted back to back allows one to discriminate between a sequential decay of the target remnant (such as a compound nucleus) or a statistical instantaneous breakup governed by phase space [29]. In view of our observation

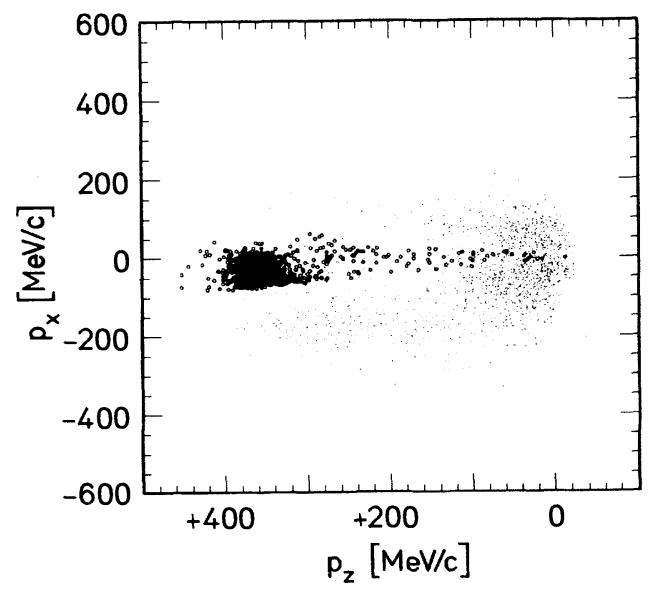

FIG. 20. Momentum distribution in the c.m. system for light fragments $3 \leq A \leq 18$ (circles) and coincident protons (points) for the condition $3^{\circ}<\vartheta_{\text {lab frag }}<11^{\circ}$ and $23^{\circ}<\vartheta_{\text {lab prot }}<78^{\circ}$. that experiment excludes the validity of the assumption that fragments come from an equilibrated system, this is a rather academic question. Nevertheless, we think it is worthwhile to make a brief comment on it.

It was found by comparing the results of a simplified compound-decay model with those of a microcanonical statistical model that both give a quite different distribution of relative velocities if applied to the decay of a $A=184, Z=74$ system with an excitation energy of 800 $\mathrm{MeV}$ and a freeze-out density of $2.2 A^{1 / 3}$. Assuming that this residue is a reasonable choice for the reaction 800 $\mathrm{MeV} /$ nucleon $\alpha+\mathrm{Th}$, the authors concluded that only an instantaneous decay is in agreement with experiment. This conjecture was subsequently doubted by Pochodzalla et al. [19], who performed sequential decay calculations with a standard compound-evaporation code for the same system and obtained quite different results, namely, an almost complete agreement of the relative velocity spectra with that of an instantaneous decay. This problem is not settled yet and seems to depend on the sequential decay code which is used. Probably, the whole issue is very difficult to settle because the key quantities of these thermal approaches, the excitation energy and freeze-out density, cannot be measured and leave usually enough room to produce quite different results. This situation worsens further if impact-parameter-dependent excitation energies and source velocities are introduced.

Since thermal models can be ruled out, it is of special importance to see whether approaches which do not rely on equilibrium assumptions can reproduce the experimental findings on fragment correlations as well.

Kinematic correlations between fragments have only been measured in this experiment and present a crucial test for the validity of any nonthermal model, such as the QMD approach. But events with two IMF's are rare in experiment as well as in our simulation. Nevertheless, the statistics of the QMD simulation is sufficient to investigate whether the general trend is reproduced. To gain statistics we have included events in which $Z=3,4$ fragments are produced, although experimentally the detection efficiency is low for these fragments. We therefore do not present theory and experiment in one graph.

In Figs. 21(a) and 21(b) we present the experimental and theoretical distributions of relative velocities between two IMF's with $A>4$ emitted at $\vartheta>23^{\circ}$ back to back $\left(\Delta \phi \approx 180^{\circ}\right)$ and the theoretical results for emission on the same side $\left(\Delta \phi \approx 0^{\circ}\right)$, respectively. We observe for backto-back emission an average relative velocity $\approx 5 \mathrm{~cm} / \mathrm{ns}$. For emission on the same side, the velocity is much smaller, around $3 \mathrm{~cm} / \mathrm{ns}$. The value we observe is about the mean value of the experimental distribution $\left(v_{\text {rel }} \approx 5\right.$ $\mathrm{cm} / \mathrm{ns})$. The reason for the difference is the Coulomb repulsion from the heavy remnant, which is always present in this reaction, and momentum conservation. Unfortunately, there are no published experimental data for emission on the same side. Clearly, in both cases the calculated relative velocities are much larger than that caused by Coulomb repulsion.

The same relative-velocity distribution for coincidences between one IMF and the heavy target residue is shown in Figs. 22(a) and 22(b). Again, we separate back-to-back 


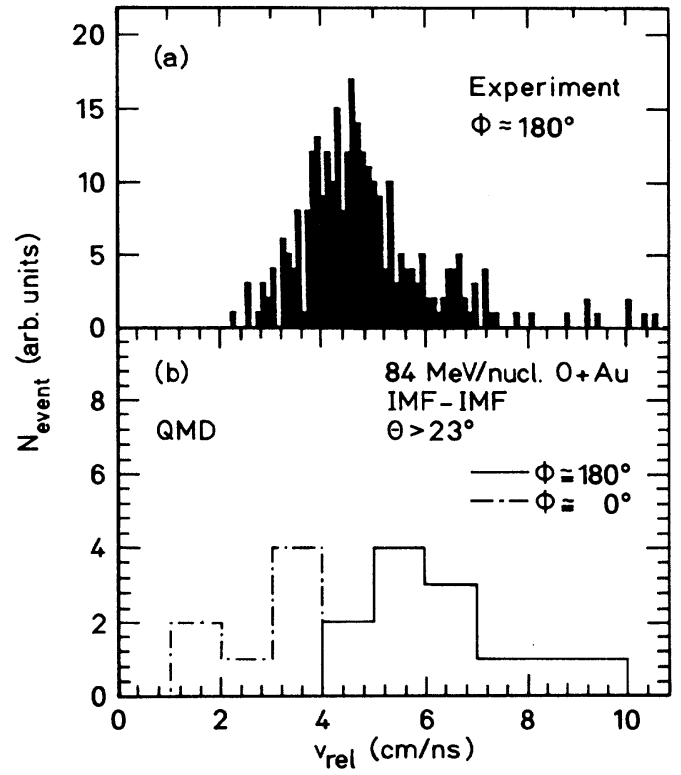

FIG. 21. Experimental and theoretical distribution of relative velocities between two coincident IMF's.

$\left(\Delta \phi \approx 180^{\circ}\right)$ and parallel emissions $\left(\Delta \phi \approx 0^{\circ}\right)$. We see a pronounced peak around $v_{\text {rel }}=3.5 \mathrm{~cm} / \mathrm{ns}$ for both cases. This velocity is slightly higher than the Coulomb barrier between both fragments. Again, the agreement with experiment is quite reasonable. The events at large relative velocities are mainly coincidences with $Z=3$ clusters, which have not been seen in the experiment as a result of thresholds.

The distribution of relative azimuthal angles

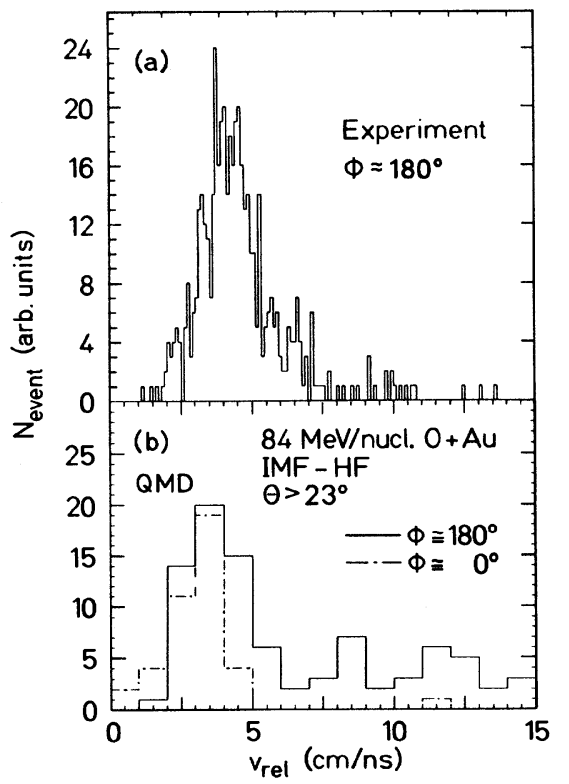

FIG. 22. Experimental and theoretical distribution of relative velocities between IMF in coincidence with a heavy residue.

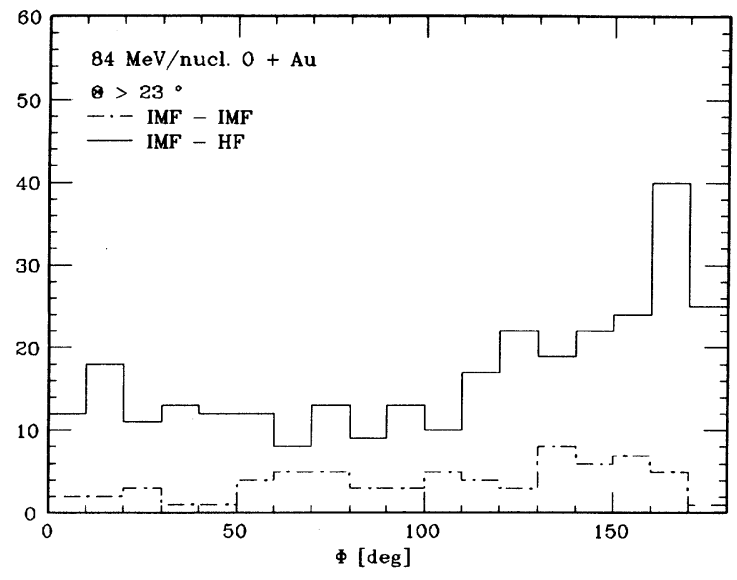

FIG. 23. Difference between the azimuthal angles of two coincident fragments.

$\Delta \phi=\phi_{1}-\phi_{2}$ between two IMF's and between one IMF and a heavy residue is shown in Fig. 23. The distribution for two IMF's is rather flat, increasing only slightly toward $\Delta \phi=180^{\circ}$. The correlation between an IMF and a heavy residue is less isotropic, but increases toward large $\Delta \phi$, as expected from momentum conservation. The emission at $\Delta \phi=180^{\circ}$ is favored because of the Coulomb repulsion when the charge of the IMF is increasing. Both distributions agree quantitatively with experiment for difference angles sufficiently large that the distribution is not spoiled by the finite granularity of the detector.

As far as coincidence and single-particle data of this reaction are published, our calculation agrees in the limit of the low statistics with the experimental findings. Thus, within one model, we can describe the highly nonthermal double-differential cross sections of protons, the fragment yields and multiplicities, and kinematic correlations between fragments. In view of our results, it seems to be rather artificial to separate protons and fragments, assuming that the first show the strong observed nonequilibrium features, whereas the fragments can be treated in thermal models such as compound evaporation of microcanonical phase-space models. Our model does not confirm the long evaporation times which are needed in sequential decay models to obtain the correct relative velocity between fragments [19]. In the QMD calculation the time between the emission of two IMF's is of the order $30 \mathrm{fm} / c$. The fragments exhibit-in our approach - the clear nonequilibrium features which have been measured.

\section{WHAT CAUSES FRAGMENTATION NEAR THE THRESHOLD?}

If the available phase space is indeed not the reason for nuclei to fragment - as it is assumed in microcanonical models $[29,19]$ - the question remains as to what causes multifragmentation. A first hint of the underlying process can be found in Fig. 7. The average longitudinal momentum of fragments is larger than that of the remnants. Thus more momentum is transferred to these frag- 
ments as compared to remnant nucleons on the average. This finding can be substantiated by inspecting the composition of the IMF's. We display in Fig. 24 the ratio of target to all nucleons entrained in these IMF's for central and peripheral collisions. We call those nucleons targetlike (respectively, projectilelike) which initially belong to target and projectile, respectively. In the figure we have suppressed those fragments which contain only projectile nucleons. We see that in central collisions around $50 \%$ of the fragments contain at least one projectile nucleon, whereas the rest contain target nucleons only. The average value of the ratio of targetlike to all nucleons contained in the IMF's is 0.88 as compared 0.99 for the target remnant.

Combining the different observations, we arrive at a reaction scenario which is very similar to the cold multifragmentation proposed a few years ago [30]. The projectile enters the target, and collisions between projectile and target nucleons take place. For the first collision the Pauli blocking is on the $25 \%$ level. The cross section is symmetric around $\vartheta=90^{\circ}$ in the nucleon-nucleon c.m. system. Hence, after the first nucleon-nucleon collision, the forward-moving scattering partner (with a momentum close to the beam momentum) is equally probable a targetlike or a projectilelike nucleon. Because of the transverse momentum transfer, the trajectories of these fast nucleons are spread over the whole nucleus. When these nucleons come to the target surface, they can either leave the target as single nucleons or they can drag along some neighboring target nucleons, thus forming a cluster. The cluster then has a momentum in between the average target remnant momentum and average participant momentum, as seen in the calculation. That roughly $50 \%$ of the IMF's contain at least one projectile nucleon presents evidence that prior to emission the projectile nucleons in the IMF's suffered about one collision (in a collision $50 \%$ of the projectile nucleons change their role as the fast nucleon with a target nucleon as a result of the isotropy of the cross section). Whether further fast nu-

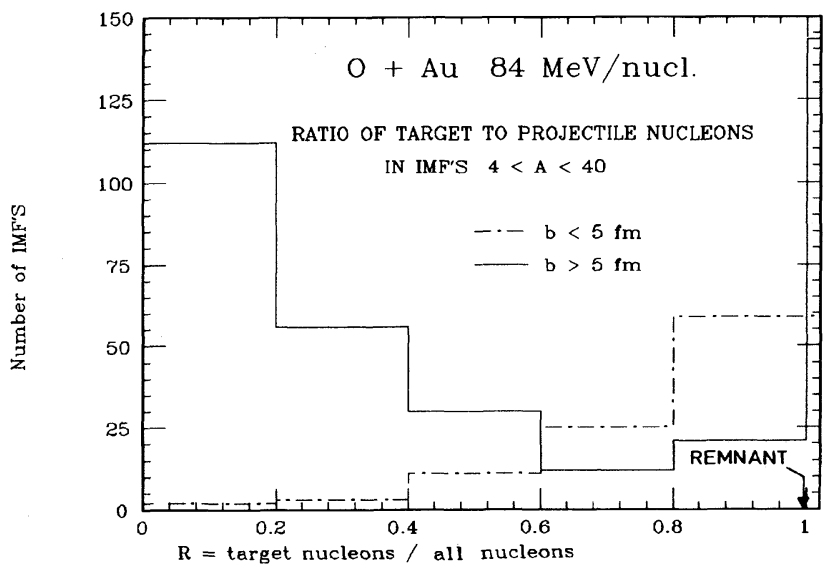

FIG. 24. Ratio of target to projectile and target nucleons contained in IMF's for two different impact-parameter bins. cleons are needed to crack the target cannot be concluded from the calculation yet.

Thus, at this low beam energy, the process which causes multifragmentation of the target nucleus is quite different from that at higher beam energies, although the slope of the mass-yield curve is almost identical. At high beam energies, $E_{\text {beam }} \gg E_{\text {Fermi }}$, the high-density wave caused by the projectile while traveling through the target causes the multifragmentation [35]. At this low energy there is no considerable increase of the density.

\section{CONCLUSIONS}

In the present paper we have studied the onset of multifragmentation in a dynamical $N$-body theory. For this purpose we have improved the QMD approach, which has been successfully applied to heavy-ion reactions at higher energies. Compared to older versions, we have incorporated the Coulomb force in the entrance channel and have further improved the stability and density profile of the initialized nuclei.

With this improved version we simulated the reaction $84 \mathrm{MeV} /$ nucleon ${ }^{18} \mathrm{O}+\mathrm{Au}$, which has been extensively investigated experimentally. Choosing the impact parameter for each simulation randomly, we can directly simulate the experiment, and no averaging procedure, such as BUU/VUU calculations, is necessary.

We observe a very strong correlation between the impact parameter and mass of the heavy residue, as well as the multiplicity of protons. Both can therefore be used-even at this low energy - as an experimental measure of the impact parameter.

We find that even in central collisions the momentum transfer is not complete and the spectra cannot be described by a single isotropically emitting source, although for a given angle the spectra have an exponential form. Rather, we observe a quite different emission pattern for different impact parameters. At each angle particles from collisions with quite different impact parameters contribute to the spectrum. The simulations give no evidence that a global equilibrium is established, neither in central collisions nor in the late stage of the reaction.

The IMF's are produced predominantly in central collisions. Their mass yield can be well described with a power law, and the slope parameter agrees well with the experimental data.

It is known experimentally and we also find [35] that the slope parameter of the mass-yield curve does not change between $80 \mathrm{MeV} /$ nucleon and $1 \mathrm{GeV} /$ nucleon. But the production mechanism for fragments is quite different at the different energies. At low bombarding energies the fragment production is compatible with the macroscopic cold fragmentation model: Fast nucleons originating from the overlap region between projectile and target traverse the cold target matter and drag along some other nucleons, forming a fragment. At these low energies nothing like a high-density wave, which causes fragmentation at higher energies, is observed in the calculations. 
For the first time we could compare our results with kinematical fragment-fragment coincidence data. These angular correlations between the fragments are well reproduced. This gives additional evidence that the range of applicability of the QMD approach can be extended to heavy systems at low energies.

\section{ACKNOWLEDGMENTS}

This work has been funded in part by the German Federal Minister for Research and Technology (BMFT) under the Contract No. 06HD710 and by the Gesellschaft für Schwerionenforschung (GSI).
*Present address: Lab. de Physique Nucléaire, 2 Rue de la Houssiniére F 44072 Naukos Cedex, France.

[1] V. Weisskopf, Phys. Rev. 52, 295 (1937).

[2] J. Gosset et al., Phys. Rev. C 16, 629 (1977).

[3] J. Aichelin, Phys. Rep. 202, 233 (1991).

[4] J. Aichelin and G. Bertsch, Phys. Rev. C 31, 1730 (1985).

[5] J. Aichelin, Phys. Rev. C 33, 537 (1986).

[6] J. Aichelin and H. Stöcker, Phys. Lett. 163B, 59 (1985).

[7] H. Kruse, B. V. Jacak, and H. Stöcker, Phys. Rev. Lett. 54, 289 (1985); H. Kruse, B. V. Jacak, J. J. Molitoris, G. D. Westfall, and H. Stöcker, Phys. Rev. C 31, 1770 (1985); J. J. Molitoris and H. Stöcker, ibid. 32, 346 (1985); Phys. Lett. 162B, 47 (1985).

[8] F. Sebille et al., Nucl. Phys. A501, 137 (1989).

[9] H. Stöcker and W. Greiner, Phys. Rep. 137, 277 (1986).

[10] R. Trockel et al., Phys. Rev. C 39, 729 (1989).

[11] R. Trockel et al., Phys. Rev. C 38, 576 (1988).

[12] R. Trockel et al., Phys. Rev. Lett. 59, 2844 (1987).

[13] R. Wada et al., Phys. Rev. Lett. 58, 1829 (1987).

[14] H. J. Rabe et al., Phys. Lett. B 196, 439 (1987).

[15] I. Bock, Diploma thesis, Heidelberg University, 1983.

[16] N. Brummund, Diploma thesis, University of Münster, 1985; Nucl. Phys. A453, 349 (1986).

[17] W. Trautmann et al., in Proceedings of the Symposium on Nuclear Dynamics and Nuclear Disassembly, Annual ACS Meeting, Dallas, 1989 (unpublished).

[18] R. Trockel, Doctoral thesis, Gesellschaft fur Schwerionenforschung, Darmstadt, F.R.G., 1987.

[19] J. Pochodzalla, Phys. Lett. B 232, 41 (1989).

[20] B. Jakobsson, in Proceedings of the 4th Bergen Workshop in Nuclear Physics, 1982 (unpublished).

[21] K. Aleklett et al., Nucl. Phys. A499, 591 (1982); Phys. Lett. B 236, 404 (1990).

[22] B. V. Jacak et al., Phys. Rev. C 35, 1751 (1987); Phys. Rev. Lett. 51, 1846 (1983).

[23] BD. J. Fields et al., Phys. Rev. C 30, 1912 (1984); Phys. Rev. Lett. 51, 1846 (1983).

[24] Y. D. Kim et al., Phys Rev. Lett. 63, 494 (1989).

[25] C. Gelbke and D. Boal, Prog. Part. Nucl. Phys. 19, 33 (1987).

[26] B. Jakobsson, Lund Report No. LUIP 8207, 1982 (unpublished).

[27] B. Jakobsson, Lund Report No. LUIP 8812, 1989 (unpublished).

[28] W. A. Friedman and W. H. Lynch, Phys. Rev. C 28, 16 (1983).

[29] D. Gross et al., Phys. Lett. B 224, 29 (1989).

[30] J. Aichelin, J. Hüfner, and R. Ibarra, Phys. Rev. C 30, 107 (1984).
[31] J. Hüfner, Phys. Rep. 125, 129 (1985).

[32] D. Gross, Rep. Prog. Phys. 53, 605 (1990).

[33] J. Bondorf et al., Nucl. Phys. A444, 460 (1985); A448, 753 (1986); Phys. Lett. 162B, 30 (1985).

[34] D. Hahn and H. Stöcker, Nucl. Phys. A476, 718 (1988).

[35] J. Aichelin and H. Stöcker, Phys. Lett. B 176, 14 (1986); A. Rosenhauer, J. Aichelin, H. Stöcker, and W. Greiner, J. Phys. (Paris) Colloq. C4, 395 (1986); J. Aichelin, A. Rosenbauer, G. Peilert, H. Stöcker, and W. Greiner, Phys. Rev. Lett. 58, 1926 (1987); J. Aichelin, G. Peilert, A. Bohnet, A. Rosenbauer, H. Stöcker, and W. Greiner, Phys. Rev. C 37, 2451 (1987); G. Peilert, A. Rosenhauer, J. Aichelin, H. Stöcker, and W. Greiner, Mod. Phys. Lett. A 3, 459 (1988); Phys. Rev. C 39, 1402 (1989).

[36] D. Boal and J. Wong, Phys. Rev. C 41, 118 (1990), and references therein; Li Zhuxia, G. Peilert, H. Stöcker, and W. Greiner, Frankfurt report, 1989 (unpublished); C. Hartnack, Li Zhuxia, L. Neise, G. Peilert, A. Rosenhauer, H. Sorge, J. Aichelin, H. Stöcker, and W. Greiner, Nucl. Phys. A495, 303 C (1989).

[37] B. Tsang, G. Bertsch, W. Lynch, and M. Tohyama, Phys. Rev. C 40, 1685 (1989).

[38] J. Aichelin, C. Hartnack, A. Bohnet, L. Zhuxia, G. Peilert, H. Stöcker, and W. Greiner, Phys. Lett. B 224, 34 (1989).

[39] P. Ring and P. Schuch, The Nuclear Many Body Problem (Springer-Verlag, Heidelberg, 1980).

[40] B. Ter Haar and R. Malfliet, Phys. Lett. B 172, 10 (1986); Phys. Rep. 149, 207 (1987).

[41] J. Cugnon, A. Lejeune, and P. Grange, Phys. Rev. C 35, 861 (1987); J. Cugnon, in Proceedings of the Nato ASI on "The Nuclear Equation of State," Peniscola, Spain, 1989 (unpublished).

[42] G. E. Brown and V. Koch, in Proceedings of the 8th High Energy Heavy Ion Study, LBL Berkeley, 1987 (unpublished); G. F. Bertsch, G. E. Brown, V. Koch, and B. Li, Nucl. Phys. A490, 745 (1988).

[43] F. De Jong, B. Ter Haar, and R. Malfliet, Phys. Lett. B 220, 485 (1989).

[44] A. Bohnet, N. Ohtsuka, J. Aichelin, R. Linden, and A. Faessler, Nucl. Phys. A494, 349 (1989).

[45] D. Vautherin and D. M. Brink, Phys. Rev. C 5, 626 (1973).

[46] P. Siemens and J. Kapusta, Phys. Rev. Lett. 43, 1486 (1979).

[47] G. Bertsch and J. Cugnon, Phys. Rev. C 24, 2514 (1981).

[48] H. A. Gustafsson et al., Mod. Phys. Lett. A 3, 1323 (1988).

[49] H. H. Gutbrod et al., Phys. Rev. C 42, 640 (1990).

[50] M. B. Tsang et al., Phys. Rev. Lett. 57, 559 (1986). 